\title{
Alleloscope: Integrative single cell analysis of allele- specific copy number alterations and chromatin accessibility in cancer
}

\section{Chi-Yun Wu}

University of Pennsylvania

Billy Lau

Stanford

Heonseok Kim

Stanford University School of Medicine

Anuja Sathe

Stanford University School of Medicine

\section{Susan M Grimes}

Stanford Genome Technology Center

Hanlee Ji

Stanford University

Nancy Zhang ( $\nabla$ nzh@wharton.upenn.edu )

University of Pennsylvania https://orcid.org/0000-0002-0880-5749

\section{Article}

Keywords: Alleloscope, somatic copy number aberrations, chromatin remodeling

Posted Date: November 4th, 2020

DOl: https://doi.org/10.21203/rs.3.rs-98536/v1

License: (9) This work is licensed under a Creative Commons Attribution 4.0 International License.

Read Full License

Version of Record: A version of this preprint was published at Nature Biotechnology on May 20th, 2021. See the published version at https://doi.org/10.1038/s41587-021-00911-w. 
1 Alleloscope: Integrative single cell analysis of allele-specific copy

2 number alterations and chromatin accessibility in cancer

3 Chi-Yun Wu ${ }^{1,2}$, Billy T. Lau ${ }^{3,4}$, Heonseok Kim³ ${ }^{3}$, Anuja Sathe ${ }^{3}$, Susan M. Grimes ${ }^{4},{ }^{*}$ Hanlee

$4 \quad$ P. Ji ${ }^{3,4}$, Nancy R. Zhang ${ }^{1,2}$

5 Institutions

$6{ }^{1}$ Graduate Group in Genomics and Computational Biology, University of Pennsylvania,

7 Philadelphia, PA

$8 \quad{ }^{2}$ Department of Statistics, University of Pennsylvania, Philadelphia, PA

9 'Division of Oncology, Department of Medicine, Stanford University School of Medicine,

10 Stanford, CA

$11{ }^{4}$ Stanford Genome Technology Center, Stanford University, Palo Alto, CA

$12{ }^{*}$ Corresponding author

13 Corresponding authors

14 Hanlee P. Ji

15 Email: genomics ji@stanford.edu

16 Nancy R. Zhang

17 Email: $\underline{\text { nzh@wharton.upenn.edu }}$ 


\section{Abstract}

20 Cancer progression is driven by both somatic copy number aberrations (CNAs) and

21 chromatin remodeling, yet little is known about the interplay between these two classes

22 of events in shaping the clonal diversity of cancers. We present Alleloscope, a method

23 for allele-specific copy number estimation that can be applied to single cell DNA and

24 ATAC sequencing data, either separately or in combination. This approach allows for

25 integrative multi-omic analysis of allele-specific copy number and chromatin accessibility

26 on the same cell. On scDNA-seq data from gastric, colorectal, and breast cancer samples,

27 with extensive validation using matched linked-read sequencing, Alleloscope finds

28 pervasive occurrence of highly complex, multi-allelic copy number aberrations, where

29 cells that carry varying allelic configurations adding to the same total copy number co-

30 evolve within a tumor. The contributions of such allele-specific events to intratumor

31 heterogeneity have been under-reported and under-studied due to the lack of methods

32 for their detection. On scATAC-seq from two basal cell carcinoma samples and a gastric

33 cancer cell line, Alleloscope detects multi-allelic copy number events and copy neutral

34 loss-of-heterozygosity, enabling the dissection of the contributions of chromosomal

35 instability and chromatin remodeling in tumor evolution. 
Cancer is a disease caused by genetic alterations and epigenetic modifications

38 which, in combination, shape the dysregulated transcriptional programming of tumor

39 cells ${ }^{1,2}$. These somatic genomic events lead to a diverse cellular population from which

40 clones with advantageous alterations proliferate and eventually metastasize ${ }^{3}$. The

41 comprehensive study of cancer requires the integrative profiling of genetic and epigenetic

42 changes at the resolution of single cells. We combined the analysis of two such genomic

43 dimensions - DNA copy number and chromatin accessibility - through massively parallel

44 single cell sequencing assays.

45 First, consider copy number aberrations (CNAs), through which we have derived much of 46 our current understanding of the relationship between genome instability and tumor

47 evolution ${ }^{4}$. Total copy number profiling, which estimates the sum of the copy numbers of

48 the two homologous chromosomes, is inadequate to characterize some types of cancer

49 genomic aberrations. Such events include the pervasively occurring copy-neutral loss of

50 heterozygosity $(\mathrm{LOH})^{5-8}$, intriguing "mirrored events"

51 cancer cells carrying amplification of one haplotype are intermingled with cancer cells

52 carrying amplification of the other haplotype, and the even more complex alterations that

53 are only detectable through allele-specific analysis ${ }^{11}$. While the importance of allele-

54 specific copy number has been emphasized in bulk DNA sequencing analysis ${ }^{5-8,11}$, most

55 single-cell CNV analysis considers only total copy number due to low per-cell coverage ${ }^{12-}$

$56{ }^{19}$. Recently, Zaccaria et al. developed $\mathrm{CHISEL}^{10}$, a method for single-cell allele-specific

57 copy number analysis, but requires externally phased haplotypes based on large 
58 reference cohorts. Despite these advances, there remain many missing details about the

59 genomic landscape of allelic imbalances when considering single cells.

60 Epigenetic modifications are also an important genomic feature of cancer. Analysis of

61 chromatin structure is feasible with a variety of methods including transposase-accessible

62 chromatin sequencing (ATAC-seq). This approach is applied either with conventional

63 bulk-based or single-cell sequencing. Subsequently, analysis of chromatin structure has

64 shown that epigenetic remodeling modulates the plasticity of cells in cancer ${ }^{20-24}$, leads to

65 stem-like properties ${ }^{25-27}$ and generates therapeutic resistance ${ }^{28-31}$. Since copy number

66 alterations involve large gains and losses of available chromatin, we expect the chromatin

67 accessibility of a region to be influenced by the changes in underlying copy number.

68 Current scATAC-seq studies estimate total copy number profiles by smoothing the read

69 coverage and normalizing the signals against a control cell population, yet this

70 appropriate control is often difficult to identify ${ }^{23,32}$. Currently, there is no method for reliable

71 total or allele-specific copy number profiling in ScATAC-seq data, and thus, how to

72 disentangle the effects of CNA and chromatin remodeling in shaping the epigenetic

73 landscape remains a challenge.

74 Addressing these challenges, we present Alleloscope, a method for allele-specific copy

75 number estimation and multiomic profiling in single cells. Alleloscope does not rely on

76 external phasing information, and can be applied to scDNA-seq data or to scATAC-seq

77 data with sample-matched bulk DNA sequencing data. To interrogate the single cell

78 landscape of allele-specific CNA, we first apply Alleloscope on scDNAseq data from four

79 gastric cancer samples, four colorectal cancer samples, and a breast cancer sample ${ }^{10,12 \text {, }}$

$80{ }^{33}$. For three of the gastrointestinal cancer samples, results are extensively validated by 
81 10x linked-read sequencing which provides accurate phasing information ${ }^{34-36}$. In these

82 datasets, Alleloscope accurately identifies $\mathrm{LOH}$ and mirrored-subclonal amplification

83 events, and finds pervasive occurrence of highly complex, multi-allelic loci, where cells

84 that carry varying allelic configurations adding to the same total copy number co-evolve

85 within a tumor. The ubiquity of such events in all three cancer types analyzed reveal that

86 they may be an important overlooked source of intratumor genetic heterogeneity.

87 Having characterized the complexity of allele-specific CNA events at single cell resolution,

88 we turn to scATAC-seq data from two basal cell carcinoma samples with paired bulk

89 whole exome sequencing data ${ }^{23}$ and a complex polyclonal gastric cancer cell line that we

90 analyzed by scDNA-seq. In these samples, we evaluate the accuracy of Alleloscope in

91 genotyping and clone assignment and demonstrate its application to the integrative

92 analysis of CNA and chromatin accessibility.

93 Results

94 Overview of Alleloscope allele-specific copy number estimation

95 First, we briefly overview Alleloscope's method for allele-specific copy number estimation

96 (Figure 1). Clone assignment and integration with peak signals in ScATAC-seq data will

97 be described later. Alleloscope relies on two types of data features: coverage, derived

98 from all reads that map to a given region, and allelic imbalance, derived from allele-

99 informative reads that cover heterozygous loci in the region. We start with some essential

100 definitions. For a given single nucleotide polymorphism (SNP) site, we refer to its mean

101 coverage across cells as bulk coverage and its mean variant allele frequency (VAF $=$ ratio

102 of alternative allele read count to total read count) across cells as its bulk VAF. Between 
103 the two parental haplotypes, we define the term "major haplotype" as the haplotype with

104 higher mean count across cells. Note that a haplotype may be the "major haplotype" of a 105 sample, but be the haplotype with lesser copy number within some cells. For each 106 individual cell $i$, in any given CNA region, we define two key parameters: (1) the major 107 haplotype proportion $\left(\theta_{i}\right)$, defined as the count of the major haplotype divided by the total 108 copy number for the region, and (2) total copy fold change $\left(\rho_{i}\right)$, defined as the ratio of the 109 total copy number of the region in the given cell relative to that in normal cells.

110 The genotyping algorithm starts by segmenting the genome into regions of homogeneous 111 allele-specific copy number using both the bulk coverage and bulk VAF profiles (Fig.1

112 Step 2). This can be achieved by multiple existing algorithms, which may be combined to 113 increase detection sensitivity, see Methods for details. In our analyses of scATAC-seq 114 data, the segmentation relied on the matched scDNA-seq data or the whole-exome 115 sequencing data, which ensures that the putative CNA regions considered for genotyping 116 are not confounded by the broad chromatin remodeling that occur in cancer.

117 Now consider each putative CNA region. An expectation-maximization (EM) based 118 algorithm is used to iteratively phase each SNP and estimate the major haplotype 119 proportion $\left(\theta_{i}\right)$ for each cell (Fig. 1, step 3). For each SNP $j$, let $I_{j} \in\{0,1\}$ be the indicator 120 of whether the reference allele of SNP $\mathrm{j}$ is a component of the major haplotype. An initial 121 estimate $\hat{I}_{j}^{(0)}$ is first derived from the bulk VAF profile. Then, in iteration $t$, Alleloscope

122 computes $\hat{\theta}_{i}^{(t)}$ by pooling counts across sites within the region, weighted by the current 123 phasing $\hat{I}_{j}^{(t)}$, then updates the estimate of $I_{j}$ based on $\hat{\theta}_{i}^{(t)}$ by pooling counts across cells.

124 The estimates of $\theta_{i}$ and $I_{j}$ usually converge within a few iterations as described in the 
125 Methods. If matched scDNA-seq data are available for a sample sequenced by scATAC126 seq, $I_{j}$ values can be estimated from scDNA-seq and then used to compute $\theta_{i}$ for each 127 cell in the scATAC-seq data, enabling integration of the two data types.

128 The estimated major haplotype proportions $\left(\hat{\theta}_{i}\right.$ 's), along with a preliminarily normalized 129 coverage statistic $\left(\tilde{\rho}_{i}\right)$, are then used to identify a set of normal cells and diploid regions

130 (Fig. 1, Step 4). This information is used to estimate an improved relative coverage fold131 change $\left(\hat{\rho}_{i}\right)$ for each cell within each CNA region. If cell $i$ 's true allele-specific copy 132 numbers are homogeneous within the given region, then its true value of $\left(\theta_{i}, \rho_{i}\right)$ should 133 belong to a set of canonical points displayed in Step 5 of Figure 1. Thus, the estimated 134 values $\left(\hat{\rho}_{i}, \hat{\theta}_{i}\right)$ are clustered across cells and associated with one of the canonical values 135 to yield the cell-level haplotype profiles for the CNV region. These cell- and region-specific 136 haplotype profiles serve as the base for clone assignment and subsequent integration 137 with peak signals in scATAC-seq data. (Fig. 4b). 
Figure 1.

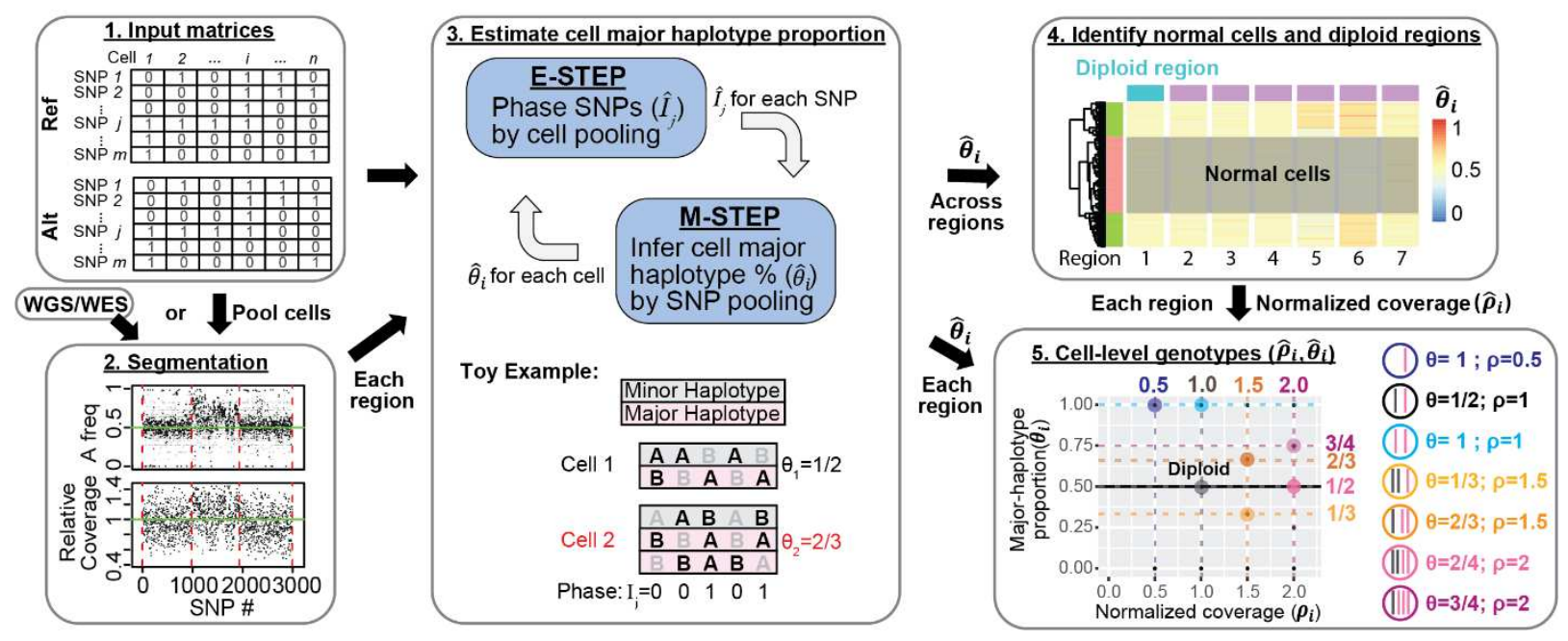

Fig. 1: Overview of allele-specific copy number estimation of single cells with Alleloscope. 1. The algorithm operates on raw read count matrices for reference allele (Ref) and alternative allele (Alt) computed from single cell DNA or ATAC sequencing. 2. First, we obtain a segmentation of the genome based on sample-matched whole genome or whole exome sequencing data using FALCON 5 . If SCDNAseq is available, cells can be pooled to derive a pseudo-bulk. 3. For each region derived from the segmentation, simultaneously phase SNPs $\left(\hat{I}_{j}\right)$ and estimate cell major haplotype proportion $\left(\hat{\theta}_{i}\right)$ by expectation maximization (EM) algorithm. Since we are focusing on only one region, the region indicator is suppressed in our notation here. In the E-step, information is pooled across cells to estimate the phasing of each SNP. In the M-step, information is pooled across all SNPs in the region are pooled to estimate the major haplotype proportion $\hat{\theta}_{i}$ for each cell. The toy example shows a scenario with two cells for a region containing 5 SNPs, with cell 2 carrying an amplification of the major haplotype (in pink). For each cell and each SNP, alleles that are observed in a sequenced read are bolded in black (we assume that only one read is observed, reflecting the sparsity of the data). The true phase $\left(I_{j}\right)$ of the SNPs and the true major haplotype proportion $\left(\hat{\theta}_{i}\right)$ are shown. 4. For region $r$ let $\left\{\hat{\theta}_{i r}\right\}$ be its estimated major haplotype proportions across cells $i$. Pool data across regions to identify candidate normal cells and candidate normal regions for computing a normalized coverage $\hat{\rho}_{i r}$ for region $r$ in cell $i$. 5 . Alleloscope assigns integer allele-specific copy numbers to each cell for each region based on the $\left(\hat{\rho}_{i r}\right.$, $\hat{\theta}_{\text {ir }}$ ) pairs. 
139 Whole genome haplotypes validate Alleloscope in scDNA-seq allele-specific copy 140 number estimation

141 First, we explore the single cell landscape of allele-specific CNAs using scDNA-seq data.

142 To validate the phasing and genotyping accuracy of Alleloscope in scDNA-seq data, we 143 used matched linked-read whole-genome sequencing data on three gastrointestinal 144 tumor samples: P5931, P6335 and P6198. Linked-read sequencing, in which one derives 145 reads from individual high molecular weight DNA molecules, provides variants that can 146 be phased into extended haplotypes covering $\mathrm{Mb}^{34-36}$. As a result, one obtains accurate,

147 Mb-scale haplotype information from cancer genome. To evaluate the accuracy of 148 phasing, we compared the haplotypes estimated by Alleloscope to the haplotypes 149 obtained from linked-read WGS. Additionally, we used the WGS haplotype to evaluate 150 the allele-specific copy number estimation for each cell and to assess the impact of 151 phasing errors on genotyping accuracy (Fig. 2a).

152 Figure $2 b$ shows the results for the gastric cancer sample from P5931, whose genome153 wide copy number profile indicates clear CNA events on four chromosomes—chr7, chr8, 154 chr20, and chr21. For each event, the scatter plots of $\left(\hat{\theta}_{i}, \hat{\rho}_{i}\right)$ estimated by Alleloscope 155 and colored by haplotype profiles, are shown in Fig. 2c. Note that the $\left(\hat{\theta}_{i}, \hat{\rho}_{i}\right)$ clusters fall 156 almost directly on top of the expected canonical values (e.g. $(1 / 2,1)$ for diploid, $(2 / 3,1.5)$

157 for 1 copy gain of major haplotype). Interestingly, chromosomes 7, 8, and 21 each show 158 subclonal clusters have differing allelic ratios but the same total copy number, which 159 would not be detectable without allele-specific estimation. We denote the major haplotype 160 of a region by " $\mathrm{M}$ ", and the minor haplotype by "m". The chromosome 7 amplification 
161 exhibits two tumor subclones with mirrored-subclonal CNAs (MMm and Mmm), each 162 subclone amplifying a different haplotype. Such a mirrored-subclonal CNA configuration

163 is also observed for the deletion on chromosome 21 ( $\mathrm{M}$ - and $\mathrm{m}-)$. The chromosome 8 164 amplification exhibits as four tumor subclones with different haplotype profiles- MMm, $165 \mathrm{Mmm}, \mathrm{MMmm}$, and MMMm.

166 We compared the phasing estimated by Alleloscope $\left(\hat{I}_{j}\right)$ against the whole genome 167 haplotypes. The phasing accuracy is $98 \%$ for the deleted region (chr21), $90 \%$ for the 168 two clonal amplifications (on chr8 and chr20), and $79 \%$ for the subclonal chr7 169 amplification (shown in the titles of the scatter plots of Fig. 2c). Moreover, we evaluated 170 the genotyping accuracy for some of the somatic alterations. Figure $2 \mathrm{~d}$ shows scatterplots 171 of $\hat{\rho}_{i}$ against major haplotype proportion computed using haplotypes derived from linked172 read sequencing $\left(\tilde{\theta}_{i}\right)$, with the same coloring as Figure $2 \mathrm{c}$. Comparing the scatterplots in 173 Figure $2 \mathrm{~d}$ to their counterparts in Figure $2 \mathrm{c}$ reveals that Alleloscope's estimated cell 174 haplotype profiles are highly concordant with those derived directly with the haplotypes 175 from linked-read WGS. Specifically, the concordance is $\sim 100 \%$ across all four events (the 176 concordance for each event is labeled in the scatter plots of Figure 2d). This shows that 177 the genotyping algorithm in Alleloscope is robust to errors in phasing (e.g. for chr7). 178 Similar analysis performed for P6335 is given in Supplementary Fig. 1. We also applied 179 CHISEL on P5931, yet it did not work well for this sample due to the low coverage 180 (Supplementary Fig. 2). 
Figure 2.

a

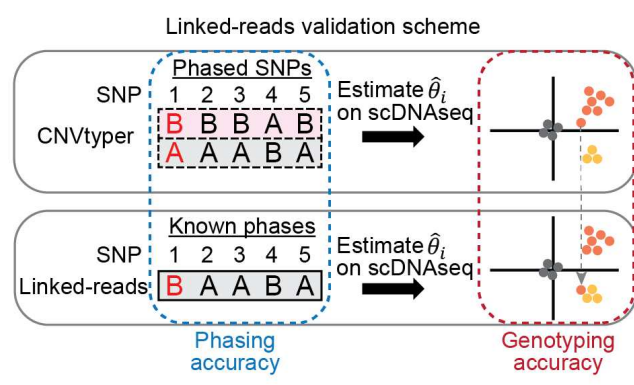

b

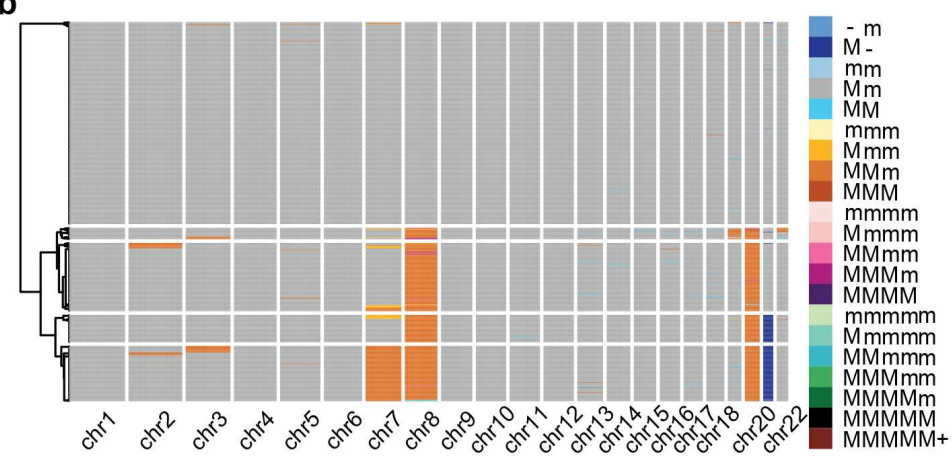

C

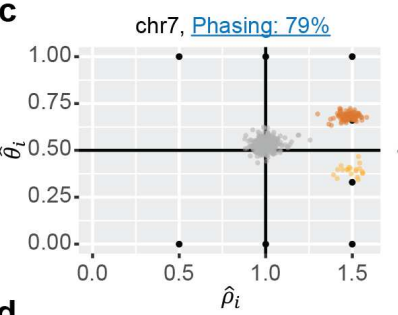

d
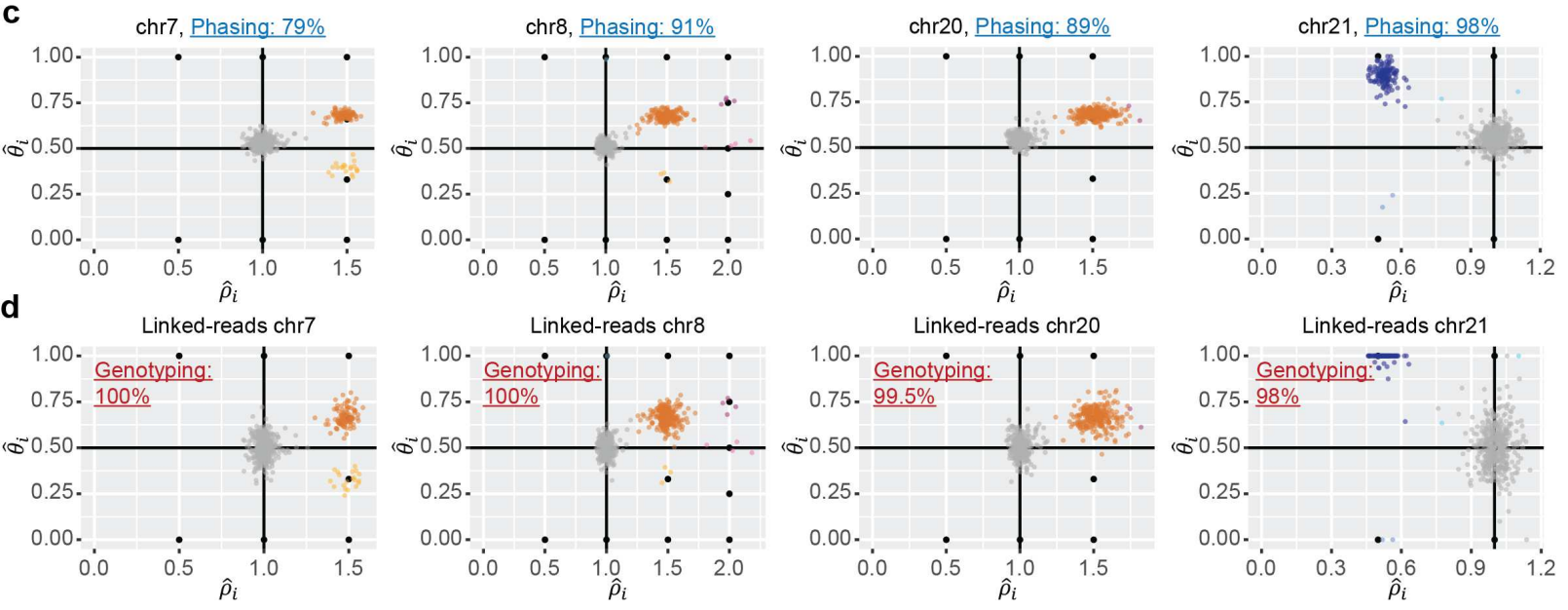

Fig. 2: Validation of the Alleloscope results on the P5931 gastric cancer patient sample and linked-reads sequencing data. (a) Illustration of the validation scheme using linked-reads sequencing data. Phasing accuracy and genotyping accuracy are used to access performance of the method. (b) Hierarchical clustering of cells in the P5931t sample based on allele-specific copy numbers given by Alleloscope, showing normal cells and 4 main clones, as well as a number of small clones marked by highly confident low-frequency mutations. M: Major haplotype, m: minor haplotype. (c) $\left(\hat{\rho}_{r i}, \hat{\theta}_{r i}\right)$ estimated by Alleloscope for four regions, colored by the inferred haplotype profile. Note that clusters fall on canonical points corresponding to discrete allele-specific copy number configurations. Phasing accuracy for each region is shown in the plot title. In the color legend, $M$ and $m$ represent the "Major haplotype" and "minor haplotype" respectively. (d) Similar to (c), with $\hat{\theta}_{i}$ estimated using known SNP phases from matched linked-reads sequencing data, colored by the haplotype profiles assigned in (c) using Alleloscope without the given phasing information. Genotyping accuracy is labeled in the plots. 
182 Since copy neutral LOH events, common in cancer genomes, can only be identified 183 through allele-specific copy number analysis. We examined the accuracy of Alleloscope 184 specifically for copy-neutral LOH events with a colorectal adenocarcinoma from P6198. 185 This tumor sample had a conventional WGS profile revealing several copy-neutral LOH 186 regions that were not evident when considering the copy number heatmap in cellranger 187 (Fig. 3a). Chromosome 5 presents an illustrative example: The bulk VAF clearly 188 separates this chromosome into two main regions, a normal region followed by a copy189 neutral LOH (Fig. 3b). Concordantly, Alleloscope reveals a cluster centered at $(\rho, \theta)=$ $190(1,1)$ corresponding to copy-neutral LOH only for the region on the right (Fig. 3b), which 191 cleanly separates the tumor cells from normal cells. Comparing to the haplotype profiles 192 derived using the haplotypes from linked-read WGS for this sample showed that the 193 accuracy of Alleloscope for copy-neutral LOH events is nearly $100 \%$ (Supplementary Fig. $1943)$. 
Figure 3.

a

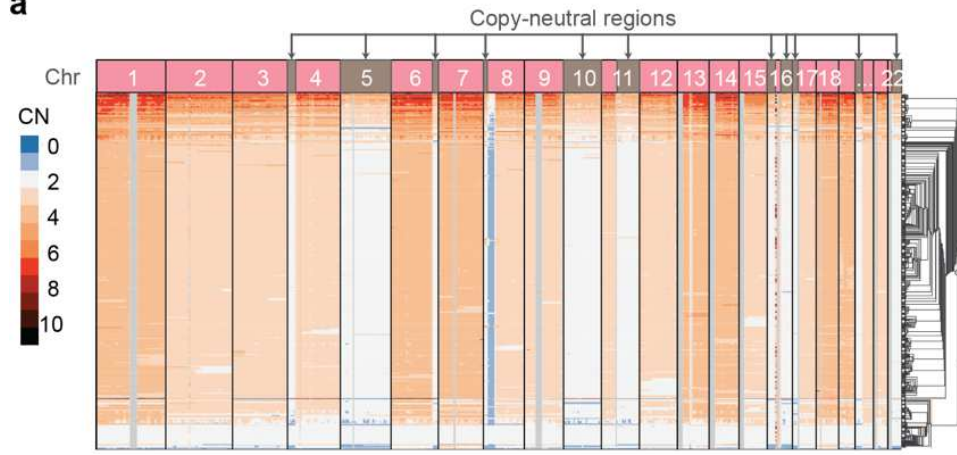

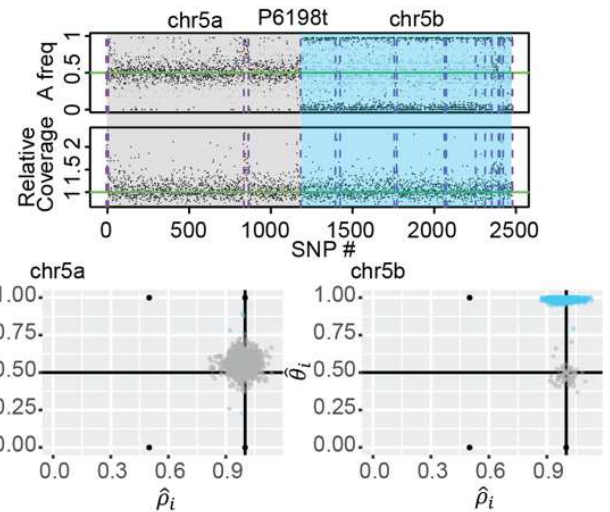

C

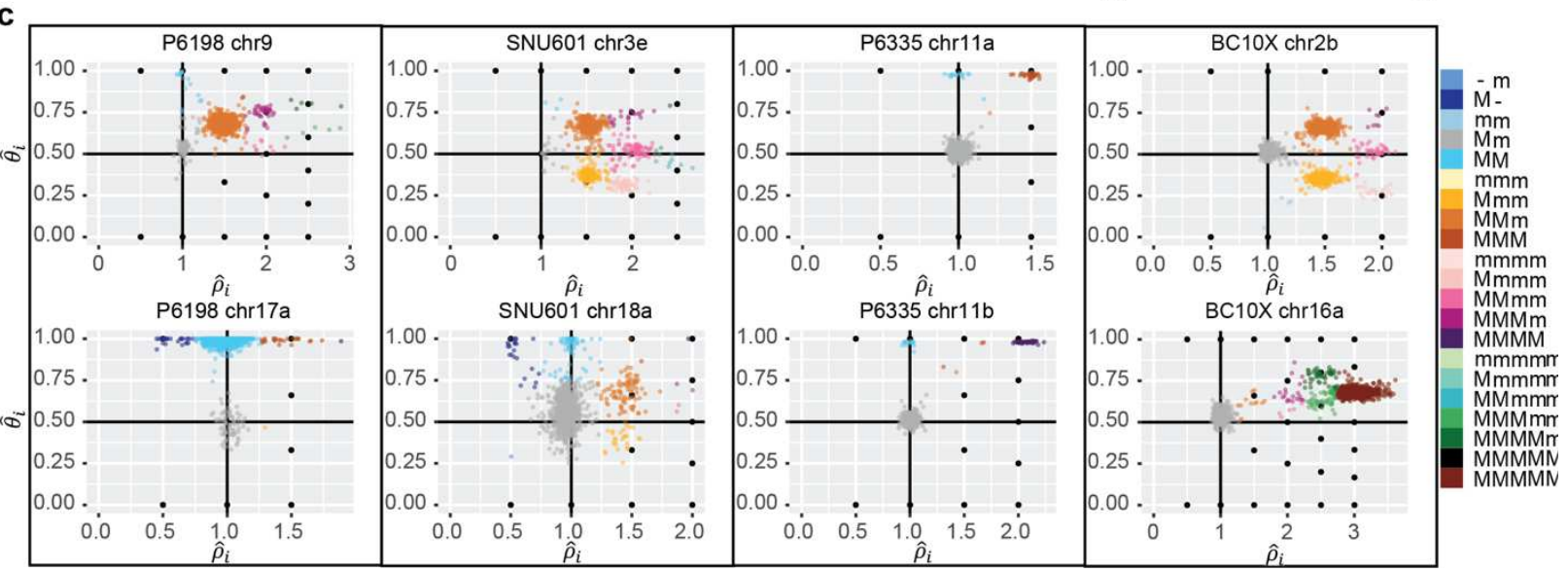

Fig. 3: Across multiple cancer types, Alleloscope detects loss-of-heterozygosity events and multi-allelic copy number aberrations, delineating complex subclonal structure which are invisible to total copy number analysis. (a) The Cell Ranger hierarchical clustering result for P6198t with copy-neutral regions labeled (total 512 cells). (b) Top: FALCON segmentation of P6198 chr5 into two regions with different allele-specific copy number profiles. Bottom: Detailed haplotype profiles of the two regions from Alleloscope, showing that the first region is diploid across cells and the second region has a loss-of-heterozygosity for a subpopulation of cells. The $a$ and $b$ following the chromosome number denote two ordered segments.

(c) Single cell allele-specific estimates $(\hat{\rho}, \hat{\theta})$, colored by assigned haplotype profiles, for select regions in the samples P6198t (metastasized colorectal cancer sample), SNU601 (gastric cancer cell line), P6335 (colorectal cancer sample), and BC10X (breast cancer cell line). In the color legend, $\mathrm{M}$ and $\mathrm{m}$ represent the "Major haplotype" and "minor haplotype" respectively. The lower-case letters following the chromosome number in the titles denote the ordered genomic segments. 
196 Alleloscope finds pervasive occurrence of polyclonal CNA regions differentiated

197 by haplotype ratios

198 The scDNA-seq samples included in this study are shown in Table 1 of Methods, with

199 detailed segmentation plots and heatmaps for genome-wide allele-specific copy number

200 profiles in supplementary figure 4-10. Across most of the samples, we observed a high

201 prevalence of complex subclonal CNAs indicated by multiple clusters of different

202 haplotype structures within a given genomic region with prototypical examples from

203 P6198, SNU601, P6335 and BC10X shown in Figure 3c. In some regions, such as

204 chromosome 9 of SNU601, 3q of SNU601, 2q and 16p of BC210x, we see as many as

205 seven subclonal clusters for a single event. In many cases there are multiple clusters

206 corresponding to the same total copy number but varying in allelic dosage. Minor

207 subclones carrying deletion of one haplotype can be easily masked by dominant

208 subclones carrying amplifications of the other haplotype in a conventional sequencing

209 analysis without the benefit of single cell resolution or an analysis that considers only

210 copy number without allelic information. Overall, the high subclonal diversity in these

211 genomic regions reveal an aspect of intratumor heterogeneity that was previously

212 undetectable.

213 Recurrent chromosomal instability events, affecting both haplotypes and producing

214 gradients in haplotype dosage, is a common theme across all samples analyzed.

215 Consider, for example, the region on chromosome 9 of P6198, which reveals 7

216 subpopulations of cells: besides the normal cell cluster and the dominant tumor cell

217 cluster with the haplotype profile MMm, there is a small cluster of cells with copy neutral

218 LOH, two small subclones at four chromosome copies and two more at five chromosome 
219 copies. This produces major haplotype ratios of $\left\{\frac{1}{2}, \frac{3}{5}, \frac{2}{3}, \frac{4}{5}, 1\right\}$ in different cells, possibly 220 conferring different fitness values. Another example of such complexity is chromosome $2213 q$ of SNU 601 and chromosome $2 q$ of BC10x, which share a similar pattern: two 222 mirrored-subclonal CNAs (MMm, mmM) at total copy number of 3, as well as mirrored223 subclonal CNAs (MMMm, MMmm, mMMM) at total copy number of 4 , producing a 224 gradient of haplotype ratios $\left\{\frac{1}{4}, \frac{1}{3}, \frac{1}{2}, \frac{2}{3}, \frac{3}{4}\right\}$. Interrogating the evolutionary route by which 225 such diversity was achieved, Alleloscope reveals that a whole genome doubling event is 226 highly likely to have taken place early in the development of BC10x and P6198, but not 227 in the development of SNU601 (see Supplementary Fig. 4\&6). Thus, the subclones at 2q 228 in BC10x and 3q in SNU601 must have evolved through different evolutionary routes: In $229 \mathrm{BC} 10 \mathrm{x}$, the early whole-genome doubling produces the cluster MMmm, from which the 230 other clusters of different haplotype profiles were most likely derived through successive 231 loss and gene conversion events. On the contrary, the clusters on $3 q$ of SNU601 were 232 most likely a result of successive amplification events starting from the normal haplotype 233 profile $\mathrm{Mm}$. The fact that different evolutionary routes, in two different cancer types (breast 234 and gastric) evolved to have such similar allelic-specific copy number patterns imply that 235 such haplotype dosage gradients may serve as an important substrate for selection in 236 tumor evolution.

237 Another recurring theme is the co-occurrence of $\mathrm{LOH}$ and amplification within the same 238 region. Often, the loss and amplification affect different haplotypes, as for chromosome $23917 p$ of P6198 and chromosome 11p, 11q of P6335. For 17p of P6198, a gene conversion 240 leading to copy-neutral LOH is most likely the early event, followed by separate loss and 241 gain events that lead to the clusters M- and MMM. Curiously, extreme instability of a 
242 chromosome region leads to clones with $\mathrm{LOH}$ of a given haplotype coexisting with clones

243 that have the same haplotype amplified. Such clones would have been undetectable in a

244 conventional bulk WGS analysis or even a single cell analysis based solely on total

245 coverage. For example, this is what occurred for SNU601's chr18a (Figure 3c). Using the

246 procedure in Figure 2a, we validated our findings of these multiallelic subclones in P6198

247 and P6335 by comparing to the paired linked-reads sequencing data. Phasing accuracy

248 is high for all LOH and amplification event types that create an allelic imbalance

249 (Supplementary Fig. 3).

250 Juxtaposition of single cell copy number and chromatin remodeling events by 251 integrative scATAC-seq analysis

252 To illustrate the integrative analysis of scATAC-seq data, we first consider two basal cell 253 carcinoma samples with matched whole-exome sequencing (WES) data ${ }^{37}$. Using the

254 matched WES data, the genome of each sample was first segmented into regions of 255 homogeneous bulk copy number (Fig. 4a, middle panel shows the segmentation for 256 SU008). Alleloscope was then applied to the scATAC-seq data to derive allele-specific 257 copy number estimates of each cell in each region. Scatterplots of $(\hat{\rho}, \hat{\theta})$ for five example 258 CNA regions and 1 control region (chr12) from SU008 are shown in Fig. 4a. For this 259 sample, peak profiles characterizing chromatin accessibility separated the cells 260 confidently into three main clusters: 308 tumor cells, 259 fibroblasts and 218 endothelial 261 cells. Since normal cells are not expected to carry broad copy number events, we 262 compared the $(\hat{\rho}, \hat{\theta})$ values of the tumor cells against those of the fibroblast and epithelial 263 cells to assess our genotyping accuracy. Density contours for each cell type are shown 
264 in the $(\hat{\rho}, \hat{\theta})$ - scatterplots (Fig. 4a). The $(\hat{\rho}, \hat{\theta})$ values clearly separate the tumor cells from

265 the normal cells for each CNV region, with the tumor cell cluster positioned at canonical 266 points, indicating that these statistics used by Alleloscope can accurately distinguish 267 amplifications and loss-of-heterozygosity events in scATAC-seq data. In particular, 268 Alleloscope differentiated the cells that carry copy neutral LOH events through shifts in 269 major haplotype proportion. Note that normal cells, which are not expected to carry broad

270 chromosome-scale CNVs, exhibit chromosome-level deviations in total coverage due to 271 broad chromatin remodeling as exemplified by the chr6b region. Furthermore, many 272 regions with no CNA signal in bulk WES data also exhibit shifts in aggregate coverage in 273 ATAC data, but with no significant difference in their $\hat{\theta}_{i}$ distribution. Thus, relying solely 274 on shifts in coverage, without complementary shifts in major haplotype proportion, would 275 lead to false positive copy number detections for scATAC-seq data.

276 By assigning allele-specific CNA profiles to single cells in scATAC-seq data, Alleloscope 277 allows the integrative analysis of chromosomal instability and chromatin remodeling as 278 follows (Figure 4b): The scATAC-seq data, paired with bulk or single-cell DNA sequencing 279 data, allows us to detect subclones. In parallel, a peak-by-cell matrix can be computed 280 following standard pipelines. Then, the subclone memberships or CNA profiles can be 281 visualized on the low-dimensional embedding of the peak matrix, and the subclones can 282 be further compared in terms of peak or transcription factor motif enrichment. Precise 283 haplotype profiles for each subclone then allow us to identify significantly 284 enriched/depleted peaks after accounting for copy number differences, thus delineating 285 events that are uniquely attributable to chromatin remodeling. 
286 Hierarchical clustering using major haplotype proportion $\hat{\theta}$ identifies the tumor cells from

287 the normal cells for both SU006 (Supplementary Fig. 11) and SU008, and clearly 288 delineates a subclone in SU008 marked by a copy-neutral LOH event on chr4a (Fig. 4c).

289 Focusing on SU008, we call the cell lineage that carries the chr4a LOH event clone-2,

290 and the other lineage clone-1. In parallel, clustering by peaks cleanly separates the tumor 291 cells from the epithelial cells and fibroblasts (Fig. 4d: left), and further, demarcates two 292 distinct clusters in the tumor cells (peaks-1 and peaks-2) (Fig. 4d: middle). What is the 293 relationship between the peaks- 1 and peaks- 2 clusters obtained from peak signals to the 294 two clones delineated by chr4a LOH? Coloring by chr4a major haplotype proportion $(\hat{\theta})$ 295 on the peaks-derived UMAP shows that the LOH in this region is carried by almost all of 296 the cells in peaks-2 but only a subset of the cells in peaks-1 (Fig. $4 \mathrm{~d}$ : middle). This can 297 also be clearly seen in the density of $\hat{\theta}$ (Fig. $4 \mathrm{~d}$ : right): While $\hat{\theta}$ is heavily concentrated 298 near 1 for peaks-2, it is bimodal for peaks-1. Since clone-1 and clone-2 are differentiated 299 by a copy-neutral event, this separation by peaks into two clusters is not driven by broad 300 differences in total copy number. Since clone-2 is split into two groups of distinct peak 301 signals, we infer that the chromatin remodeling underlying the divergence of the peaks-2 302 cells must have occurred in the clone-2 lineage, after the chr4a LOH event (Fig. 4e). In 303 this way, Alleloscope analysis of this scATAC-seq data set allowed us to overlay two 304 subpopulations defined by peak signals with two subpopulations defined by a subclonal 305 copy-neutral LOH, and infer their temporal order. 
Figure 4.
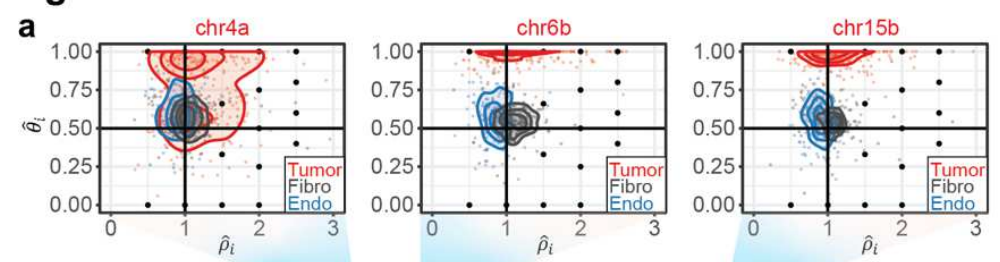

HMM segmentation on WES data

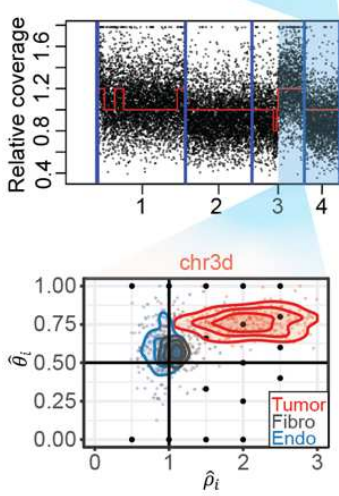

W 5 网

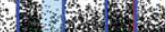

1. 12

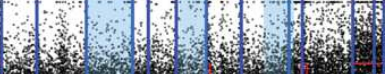

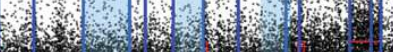

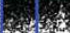

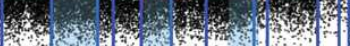
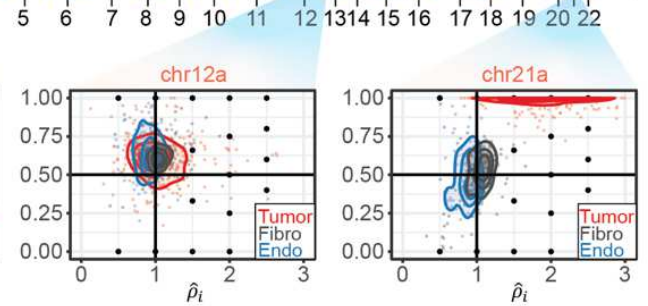

C Cell $\quad$ Type
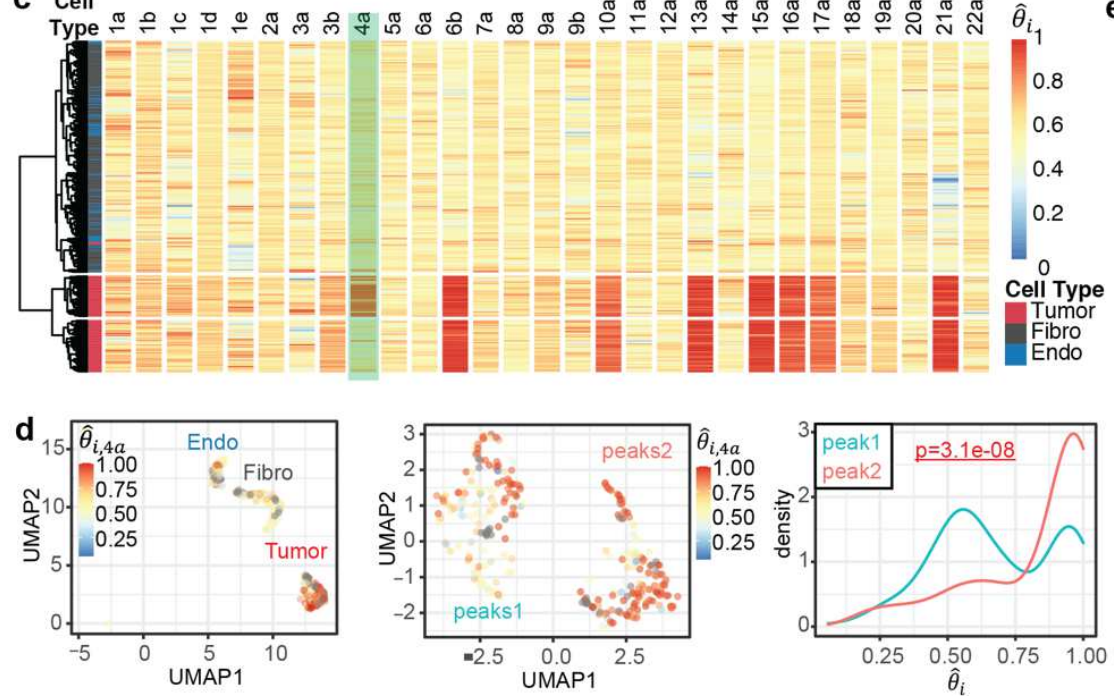

e

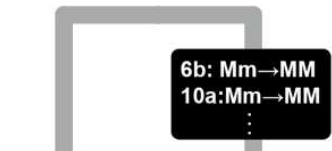

Normal

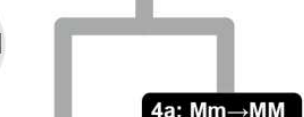

Clone1

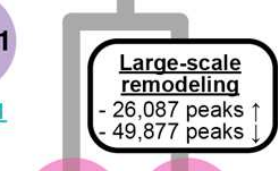

Clone2 Clone2

Peaks1 Peaks2

Fig. 4: Alleloscope multiomic analysis of scATAC-seq data of a basal cell carcinoma sample $\left(\mathbf{S U 0 0 8 ^ { 2 3 }}\right)$. (a) Genotype profiles for six example regions for cells in scATAC-seq data. The regions are taken from segmentation of matched whole exome sequencing (WES) data. Each dot represents a cellspecific $\left(\hat{\rho}_{i}, \hat{\theta}_{i}\right)$ pair. Cells are colored by annotation derived from peak signals ${ }^{23}$, Tumor: tumor cells, Fibro: fibroblasts, Endo: Endothelial cells]. Density contours are computed for each cell type (tumor, fibroblasts, endothelial) separately and shown by color on the plot. The lower-case letters following the chromosome number in the titles denote the ordered genomic segments. (b) Pipeline for multi-omics analysis integrating allele-specific copy number estimates and chromatin accessibility peak signals on ATAC-seq data. (c) Hierarchical clustering of cells by major haplotype proportion $(\hat{\theta})$ allows the separation of tumor cells from normal cells, as well as the differentiation of a subclone within the tumor cells. The marker region on chr4a separating the two tumor subclones is highlighted. (d) Integrated visualization of chr4a major haplotype proportion $\left(\hat{\theta}_{i}\right)$ and genome-wide peak profile. Left: UMAP projection of the 788 cells in the dataset by their genome-wide peak profile, colored by $\hat{\theta}_{i}$. The cell type annotation (endothelial, fibroblasts, and tumor cells) is labeled in the plot. Middle: UMAP projection of only the 308 tumor cells by their genome-wide peak profile shows two well-separated clusters: peaks 1 and peaks2. Right: Density of $\hat{\theta}_{i}$ values for the peaks1 and peaks2 subpopulations. (e) Intratumor 
heterogeneity of SU008 is shaped by a subclonal LOH of chr4a followed by subsequent genome-wide chromatin remodeling leading to three subpopulations: Clone 1 which does not carry the chr4a LOH (peaks cluster 1), Clone 2 carrying the chr4a LOH (peaks cluster 1), and remodeled clone 2 (peaks cluster 2).

307 Integrative analysis of clonal evolution and altered chromatin accessibility for a 308 complex polyclonal gastric cancer cell line

309 The gastric cancer cell line SNU601 exhibits complex subclonal structure, as evidenced

310 by multiple multiallelic CNA regions (chr3e and chr18a are shown in Figure 3c). In addition

311 to scDNA-seq, we also performed scATAC-seq on this sample to profile the chromatin

312 accessibility of 3,515 cells at mean coverage of 73,845 fragments per cell. This allows us

313 to compare the allele-specific copy number profiles obtained by scATAC-seq with those

314 given by scDNA-seq and integrate the two data types in a multi-omic characterization of

315 this complex tumor.

316 First, we segmented the genome and estimated the allele-specific copy number profiles

317 of single cells at each segment for both the scATAC-seq and scDNA-seq data, following

318 the procedure in Figure 1 with some modifications due to the lack of normal cells to use

319 as control for this sample (see methods). Figure 5a shows the relative total coverage,

320 pooled across cells from scDNA-seq. Figure $5 b$ shows $(\hat{\rho}, \hat{\theta})$-scatterplots for five example

321 CNA regions in scDNA-seq and scATAC-seq. Compared to the scATAC-seq data, the

322 scDNA-seq data has about 8-fold higher total read coverage and 7-fold higher

323 heterozygous site coverage per cell. Thus, while subclones corresponding to distinct

324 haplotype profiles are cleanly separated in the scDNA-seq data, they are much more

325 diffuse in the scATAC-seq data. Yet, cluster positions in scATAC-seq roughly match those 
326 in scDNA-seq. As expected, the $(\hat{\rho}, \hat{\theta})$-scatterplots reveal the high level of chromosomal

327 instability in this sample, with each region exhibiting multiple clusters of different

328 haplotype structures that indicate the existence of subclones carrying mirrored events

329 and, for some regions, the variation of haplotype dosage over a gradient across cells.

330 Figure $5 \mathrm{c}$ shows the hierarchical clustering of cells from scDNA-seq based on their allele-

331 specific copy number profiles, revealing the subclonal structure and the co-segregating

332 CNA events that mark each subclone. For each cell in each region, Alleloscope also

333 produces a confidence score for its assignment to different haplotype profiles

334 (Supplementary Fig. 12). Based on visual examination of the confidence scores at the

335 marker regions, we identified 6 subclones for further investigation (Clones 1-6 labeled at

336 the right of the heatmap). The allele-specific copy number profiles allow us to manually

337 reconstruct the probable evolutionary tree relating these 6 clones under the following

338 three rules:

339 (1) Parsimony: The tree with the least number of copy number events is preferred.

340 (2) Monotonicity: For a multi-allelic region with escalating amplifications (e.g. Mm, MMm,

$341 \mathrm{MMMm}$ ), the haplotype structures were produced in a monotonic order (e.g. $\mathrm{Mm} \rightarrow$

$342 \mathrm{MMm} \rightarrow \mathrm{MMMm}$ ) unless a genome doubling event occurred.

343 (3) Irreversibility of LOH: Once a cell completely loses an allele (i.e. copy number of that

344 allele becomes 0 ), it can no longer gain it back.

345 The evolutionary tree, thus derived, is shown in Figure 6b. The mirrored-subclonal 346 amplifications on chr3q, the deletion on chr4p, and the multiallelic amplification on chr20q 
347 allowed us to infer the early separation of clones 3-6 from clones 1-2. Subclones 3-6 are 348 confidently delineated by further amplifications on chr3q, chr20q, chr11, chr13, and chr17.

349 Note that high chromosomal instability led to concurrent gains of $1 q$ and $7 p$ in both the

350 Clone 1-2 and Clone 3-6 lineages. We also observed a large number of low-frequency

351 but high-confidence CNA events indicating that ongoing chromosomal instability in this

352 population is spawning new sporadic subclones that have not had the chance to expand.

353 We now turn to scATAC-seq data, focusing on the 10 marker regions which, together,

354 distinguish Clones 1-6: chr1b, 3b-d, 4b, 7a, 11b, 13b, and 20b-c. The $(\hat{\rho}, \hat{\theta})$ values 355 computed by Alleloscope allows us to directly assign allele-specific copy number profiles

356 to each cell for each region, as well as subclone labels to each cell, with posterior 357 confidence score. The subclone assignment utilizes a Bayesian mixture model that pools 358 information across the 10 marker regions. Despite the low accuracy in per-region 359 genotyping, when information is pooled across the 10 marker regions, $81.6 \%$ of the 2,753 360 cells after filtering can be assigned to a subclone with $>95 \%$ posterior confidence 361 (Supplementary Fig. 13, the number of ATAC cells confidently assigned to each clone 362 are shown in Figure 6a.). These subclone assignments for each cell, and cell-level 363 haplotype profiles for each region, can now be integrated with peak-level signals. 
Figure 5.

a

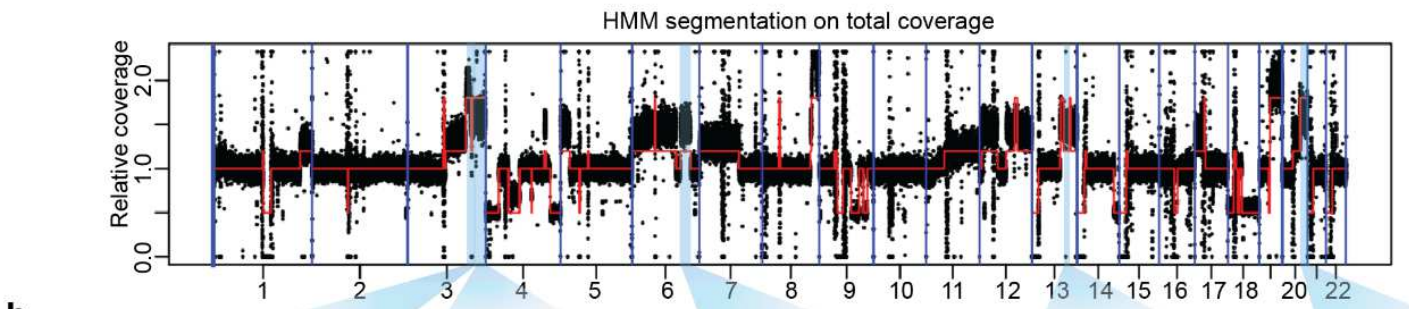

b
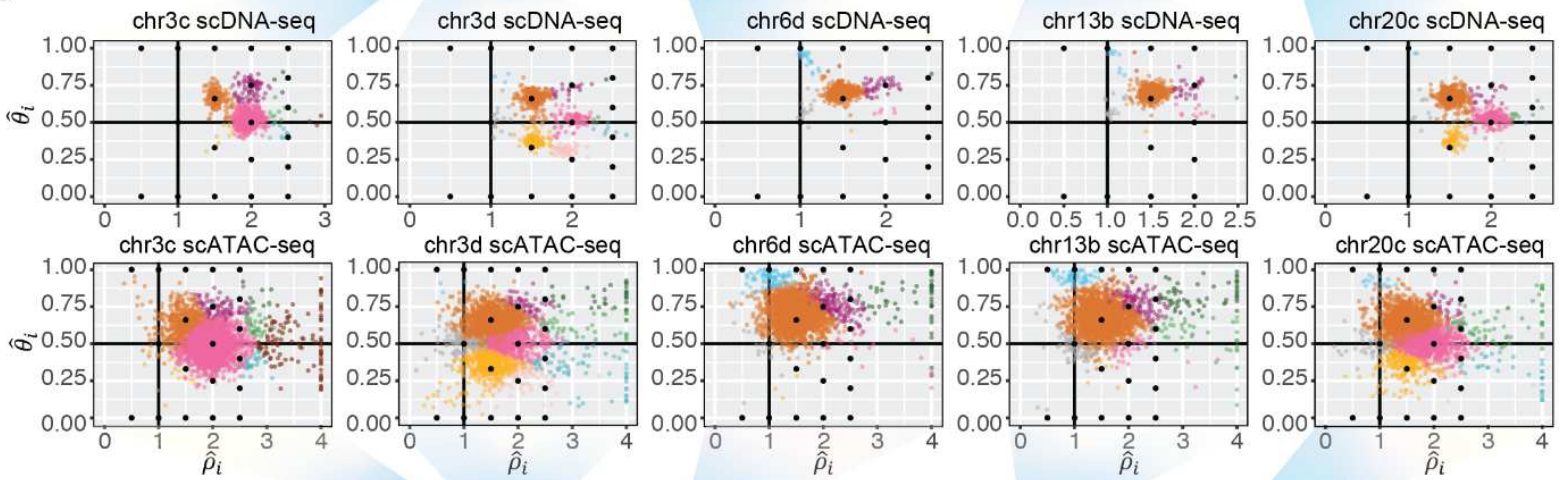

C

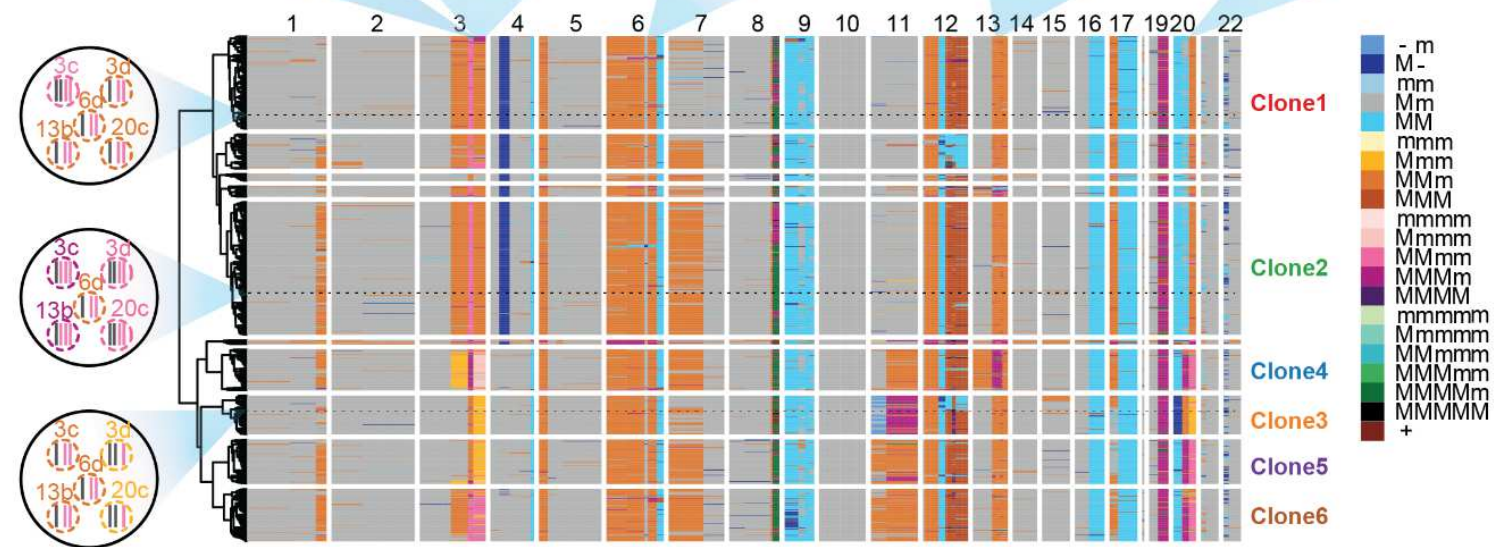

Fig. 5: Alleloscope analysis of scDNA-seq and scATAC-seq data reveals complex subclonal heterogeneity in the SNU601 gastric cancer cell line. (a) Genome segmentation using HMM on the pooled total coverage profile computed from scDNA-seq data.

(b) Single cell allele-specific copy number profiles $(\hat{\theta}, \hat{\rho})$ for five regions in scDNA-seq and scATAC-seq data. Cells are colored by haplotype profiles according to legend in Figure 5c.

(c) Tumor subclones revealed by hierarchical clustering of allele-specific copy number profiles from the scDNA-seq data. Genotypes of the five regions shown in Figure 5b, for three example cells, are shown in the left. The haplotype structures for the 5 regions in Figure $5 \mathrm{~b}$ of three cells randomly chosen from Clone 1, 2, and 3, are shown to the left of the heatmap. In the color legend, $\mathrm{M}$ and $\mathrm{m}$ represent the "Major haplotype" and "minor haplotype" respectively. The six clones selected for downstream analysis in scATAC-seq data are labeled in the plot. 
365 Following the scheme in Figure 4b, we computed the Uniform Manifold Approximation 366 and Projection (UMAP) coordinates for the scATAC-seq cells based on their peak profiles,

367 which gives a two-dimensional visualization of the geometry of the chromatin accessibility 368 landscape of this sample (Fig. 6a). UMAP scatterplots colored by clone assignment show 369 that the 6 clones exhibit marked differences in their chromatin accessibility profiles (Fig. 370 6a): While Clone 1 and Clone 2 are concentrated at the top half of the UMAP, Clones 33715 are positioned almost exclusively at the bottom half. Clone 6 , which exhibits more 372 variance, is also significantly enriched at the bottom half of the UMAP. Among Clones 33735 , Clone 3 has a distinct chromatin accessibility profile that is mostly concentrated at the 374 bottom tip, Clone 4 is positioned higher, while Clone 5 contains cells that are similar to 375 both clones 3 and 4 . We expect some of these peak-level differences to be driven by 376 CNAs.

377 To delineate the peaks that differ between clones, and to distinguish peak differences 378 that are not accountable by CNAs, we identified differential accessibility peaks (DAPs) 379 across each split of the tree (Fig. 6b) by performing pairwise Chi-square tests for peak 380 enrichment between the cell populations on the two branches. The DAPs are categorized 381 into two groups -1. DAPs lying in CNA regions for which the direction of change aligns 382 with the direction of change of DNA coverage, and 2. DAPs not in CNA regions and DAPs 383 in CNA regions that don't align in directionality of change with DNA coverage. The number 384 of DAPs in both groups are shown along each branch (Fig. 6b). For the smaller subclones 385 (Clone 3,4,5), low coverage limits the detection power and thus limits the DAP counts in 386 both categories. Yet, juxtaposing DAP and CNA events along the tumor phylogeny yields 387 insights: Along most lineages, a significant proportion of DAPs are attributable to CNAs 
388 (p-values shown along each branch), and CNA events drive a substantial $36.3 \%$ of all of 389 the DAPs identified. This argues for the importance of CNAs as a mechanism underlying 390 subclonal differences in chromatin accessibility in this tumor.

391 Nevertheless, along some branches we find a large number of DAPs not attributable to 392 broad CNAs, and thus must be due to other mechanisms. Two example DAPs of this 393 latter category are shown as insets in Figure 6b, with full list given in Supplementary Table 394 1. The first example is a peak at the transcription start site (TSS) of the REC8 gene, which 395 is located on chr14 where no apparent CNAs were observed across the six major 396 subclones. The TSS of REC8 is open in clones 3-6 but closed in clones 1-2 (p397 value $<0.0001)$. REC8 is a gene encoding a meiosis-specific cohesion component that is 398 normally suppressed in mitotic proliferation, and its role in cancer has recently gained 399 increasing attention and controversy: While Yu et al. ${ }^{38}$ found the expression of this gene 400 to suppress tumorigenicity in a gastric cancer cell line, McFarlane et al. ${ }^{39}$ postulated that 401 it may be broadly activated in some cancers where it generates LOH by reductional 402 segregation. The opening of the TSS of REC8, stably maintained in Clones 3-6, suggests 403 that meiotic processes may underlie the increased chromosomal instability of this 404 multiclonal lineage. The second example is a peak at the TSS of the WWOX gene, located 405 on chr16, which is significantly depleted in Clone 3 ( $p$-value<0.0001). Although chr16 has 406 LOH across all tumor cells, there are no detectable subclonal differences, and thus we 407 don't expect the decrease in accessibility at WWOX for subclone 3 to be due to a large 408 copy number event. Since WWOX is a well-known tumor suppressor whose down409 regulation is associated with more advanced tumors ${ }^{40,41}$, its decrease in accessibility 410 suggests a more aggressive phenotype for Clone 3. Overall, these two examples show 
411 how Alleloscope can be used to dissect the roles of CNA and chromatin-level changes in 412 the identification of gene targets for follow-up study. 


\section{Figure 6.}

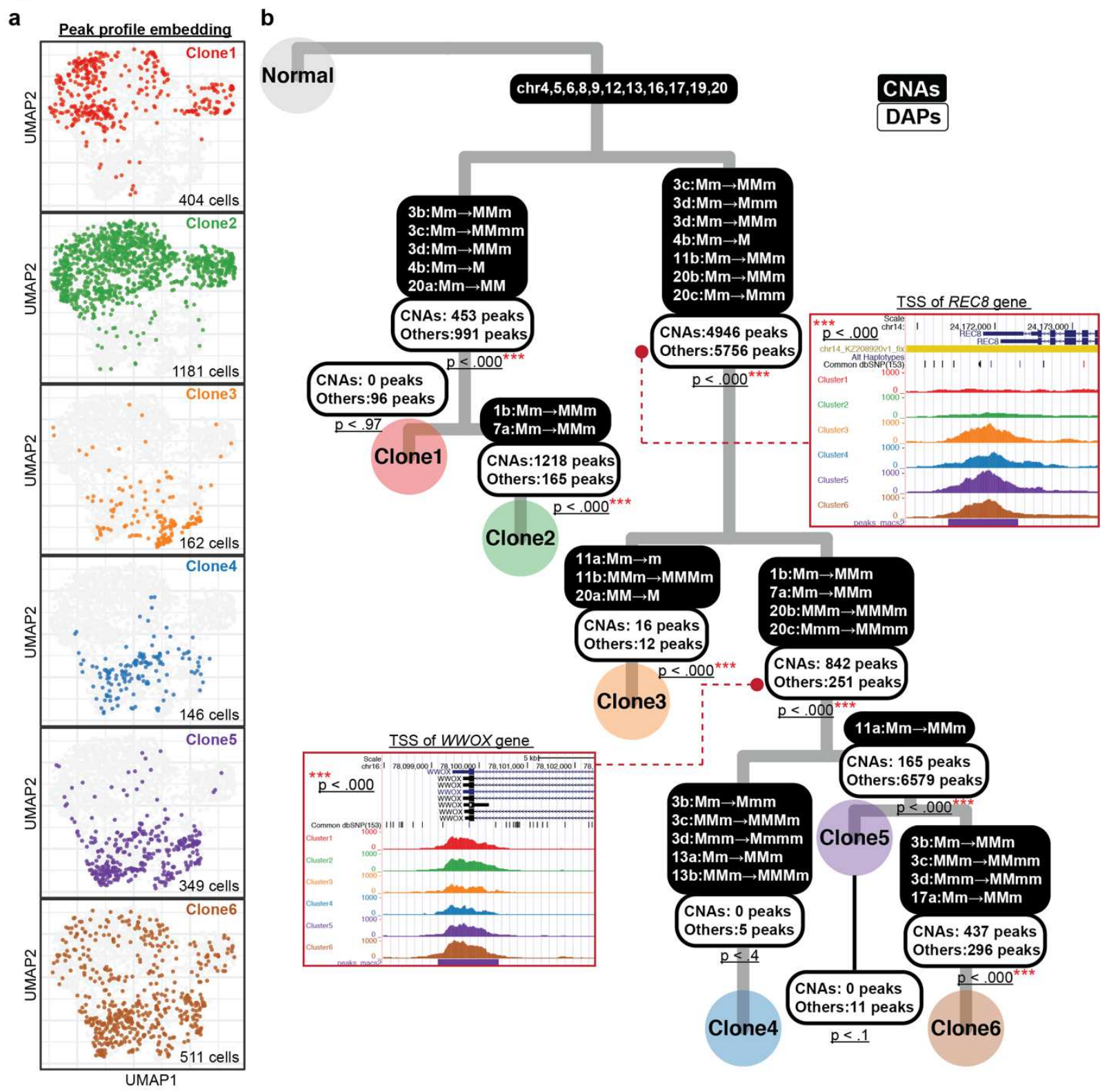

Fig. 6: Integrative analysis of allele-specific copy number and chromatin accessibility for SNU601 ATAC sequencing data. (a) UMAP projection of genome-wide sCATAC-seq peak profile on 2,753 cells. The same group of cells were clustered into one of the six subclones based on their allele-specific copy number profiles across the 10 selected regions. Cells in different subclones are labeled with different colors, using the same color scheme as that for the subclone labels in Fig. 4c. The number of cells colored in each UMAP is shown at the bottom-right corners.

(b) A highly probable lineage history of SNU601, with copy number alterations (CNAs) and differential accessibility peaks (DAPs) marked along each branch. P-values of the tests for association between DAPs and CNAs are shown along each branch. For two example DAP genes, pooled peak signals for each subclone are shown as inset plots. 


\section{Discussion}

415 Despite the recent advances in the application of single cell sequencing to cancer, we are

416 still far from understanding the diversity of genomes that are undergoing selection at the

417 single cell level. Notably, little is yet known about the intratumor diversity of allelic

418 configurations within CNV regions, and to what extent the diversity of cells in chromatin

419 accessibility can be attributed to diversity in allele-specific copy number. We presented

420 Alleloscope, a new method for allele-specific copy number estimation that can be applied

421 to single cell DNA and ATAC sequencing data (separately or in combination). First, on

422 scDNA-seq data of 9 samples from 3 different tumor types, with phasing validation by

423 linked-read sequencing on three samples, Alleloscope revealed an unprecedented level

424 of allelic heterogeneity within hypermutable CNA regions. In these regions, subclones

425 reside on a gradient of allelic ratios that is unobservable in total copy number analysis. In

426 simple cases, these hypermutable regions contain mirrored subclones, as previously

427 identified $^{9,10}$, but are often much more complex. We observed multiple instances of

428 recurrent CNA events, some verified by linked read sequencing, where the same region

429 is mutated multiple times during the evolution of the tumor, arriving at the same haplotype

430 profile in distinct clones. In accordance with the findings in Watkins et al. ${ }^{42}$, we found

431 using Alleloscope that chromosomal instability drives the formation of subclones not only

432 in primary tumors but also after metastasis.

433 Having established the allelic complexity of CNAs at single cell resolution, we next applied

434 Alleloscope to scATAC-seq data, thus enabling the combined study of clonal evolution

435 and chromatin accessibility. First, we considered the analysis of a public data set

436 consisting of two basal cell carcinoma samples, for which matched bulk whole-exome 
437 sequencing data was used for initial genome segmentation upon which single cell CNA 438 genotyping was then conducted in the scATAC-seq data. Here we showed that 439 Alleloscope can detect amplifications, deletions, and copy-neutral LOH events accurately 440 in scATAC-seq data, and was able to find a subclone delineated by a copy-neutral LOH 441 event. Juxtaposing this subclone assignment with peak signals allowed us to detect a 442 wave of genome-wide chromatin remodeling in the lineage carrying the $\mathrm{LOH}$. Next, we 443 applied Alleloscope to a complex polyclonal gastric cancer cell line with matched scDNA444 seq data. We found, by overlaying peak signals with subclones delineated by allele445 specific copy number estimates, that much of the intratumor heterogeneity in chromatin 446 accessibility can be attributed to CNAs. Focusing on subclone-enriched peaks outside of 447 CNA regions allowed the prioritization of genes for downstream follow-up.

448 Alleloscope can potentially be applied to the integration of single cell data of other 449 modalities, for example scATAC-seq and scRNA-seq data, to investigate the relationships 450 between clonal evolution, chromatin remodeling, and transcriptome. To facilitate 451 experimental design for single cell omics sequencing protocols, we investigated the 452 performance of Alleloscope under different scenarios (number of cells, total per cell 453 coverage, and total coverage at heterozygous SNP sites), see Supplementary Methods. 454 As expected, accuracy is a function of all three quantities (Supplementary Fig. 14). 455 Coverage at heterozygous SNP sites is especially important for scRNA-seq and scATAC456 seq data, for which shifts in total coverage is an unreliable proxy for underlying DNA copy 457 number. For scATAC-seq, the lower heterozygosity within peak regions led to lower 458 number of reads mapping to heterozygous loci as compared to scDNA-seq, and this 459 resulted in noisier subclone detection. Most of the current scRNA-seq technologies only 
460 sequence either the $3^{\prime}$ or 5 ' end of the mRNA transcripts, which limits the number of

461 heterozygous SNP sites covered by reads. The latest developments in single cell long

462 read sequencing ${ }^{43-45}$ and single cell multimodal sequencing ${ }^{46}$ herald new analysis

463 opportunities with this method.

464 Methods

465 ScDNA-seq Data Sets and Pre-processing

466 Table 1 summaries the nine 10x scDNA-seq samples analyzed in this study:

\begin{tabular}{llllllll} 
Sample & $\begin{array}{l}\text { Cancer } \\
\text { type }\end{array}$ & Source & $\begin{array}{l}\text { Paired } \\
\text { normal }\end{array}$ & $\begin{array}{l}\text { Linked } \\
\text {-reads }\end{array}$ & $\begin{array}{l}\text { Coverage } \\
\text { per cell }\end{array}$ & $\begin{array}{l}\text { Cell } \\
\text { number }\end{array}$ & Ref \\
\hline P5846 & Gastric & Primary tissue & Yes & No & 454,806 & 510 & 33 \\
P5847 & Gastric & Primary tissue & Yes & No & 422,134 & 715 & 33 \\
P5915 & Colorectal & Liver meta & Yes & No & 126,2629 & 233 & 33 \\
P5931 & Gastric & Primary tissue & Yes & Yes & 730,932 & 796 & 12 \\
P6198 & Colorectal & Liver meta & Yes & Yes & 532,343 & 2,271 & 33 \\
P6335 & Colorectal & Omentum meta & No & Yes & 564,058 & 953 & 33 \\
P6461 & Colorectal & Liver meta & Yes & No & 483,524 & 1,242 & 33 \\
SNU601 & Gastric & Ascites meta & No & No & 565,648 & 1,531 & 12 \\
BC10x & Breast & Primary tissue & No & No & 781,506 & 1,916 & 10
\end{tabular}

467 The Cell Ranger DNA pipeline (https://support.10xgenomics.com/single-cell468 dna/software/) automates sample demultiplexing, read alignment, CNA calling and

469 visualization. We first applied the tool to process the sequencing data (beta version:

$4706002.16 .0)$ using the GRCh38 reference genome. The output bam files from the tool

471 contain all information for later analysis. If the tumor samples had a matched normal

472 sample, the GATK HaplotypeCaller was used to reliably call heterozygous SNPs on the

473 matched normal samples. Otherwise, SNPs were retrieved on the tumor sample

474 themselves. Next, we applied VarTrix, a software tool for extracting single cell variant

475 information from the 10x barcoded bam files (https://github.com/10XGenomics/vartrix), to 
476 efficiently generate two SNP-by-cell matrices for both reference alleles and alternative

477 alleles of the SNPs called in the previous step.

478 To include high-quality SNPs in the later analysis, we filtered out the SNPs with $<5$ reads

479 for P5846 and P5847, <10 reads for P5915 and P5931, <15 reads for P6335 and P6461,

$480<20$ reads for P6198 and SNU601, and $<40$ for BC10X samples based on the number of

481 SNP detected for each sample. Additionally, SNPs located in the regions of repetitive

482 sequences such as centromeres and telomeres were excluded. To exclude cells that

483 might undergo apoptosis or cell cycles, the cells labeled noisy from the metadata output

484 by the Cell Ranger tool were excluded.

485 Single-cell ATAC Data sets, Sequencing and Preprocessing

486 Table 2 summaries the scATAC-seq samples analyzed in this study:

\begin{tabular}{lllllll} 
Sample & $\begin{array}{l}\text { Cancer } \\
\text { type }\end{array}$ & Source & $\begin{array}{l}\text { Matched } \\
\text { DNA }\end{array}$ & $\begin{array}{l}\text { Coverage } \\
\text { per cell }\end{array}$ & $\begin{array}{l}\text { Cell } \\
\text { number }\end{array}$ & Ref \\
\hline SU006 & $\begin{array}{l}\text { Basal cell } \\
\text { carcinoma }\end{array}$ & Primary tissue & Yes & 41,368 & 2771 & 23 \\
SU008 & $\begin{array}{l}\text { Basal cell } \\
\text { carcinoma }\end{array}$ & Primary tissue & Yes & 36,057 & 788 & 23 \\
SNU601 & $\begin{array}{l}\text { Gastric } \\
\text { Ascites meta }\end{array}$ & Yes & 73,845 & 3614 & -
\end{tabular}

488 The scATAC-seq dataset for the SNU601 sample was generated in this study. About 489400,000 cells were washed with RPMI media and centrifuged ( $400 \mathrm{~g}$ for 5 min at $4^{\circ} \mathrm{C}$ )

490 twice. The supernatant was removed and chilled PBS $+0.04 \%$ BSA solution was added.

491 The resuspended pellet was added to a $2 \mathrm{ml}$ microcentrifuge tube and centrifuged $(400 \mathrm{~g}$

492 for $5 \mathrm{~min}$ at $4^{\circ} \mathrm{C}$ ). After removing the supernatant without disrupting the pellet, $100 \mu \mathrm{L}$ of

493 chilled Lysis Buffer (10 mM Tris- $\mathrm{HCl}$ (pH 7.4), $10 \mathrm{mM} \mathrm{NaCl}, 3 \mathrm{mM} \mathrm{MgCl}$, $1 \%$ BSA, $0.1 \%$ 
494 Nonidet P40 Substitute, $0.1 \%$ Tween-20 and $0.01 \%$ digitonin) was added and carefully 495 mixed 10 times. The tube was incubated on ice for $7 \mathrm{~min}$. After incubation, $1 \mathrm{~mL}$ of chilled 496 Wash Buffer (10 mM Tris- $\mathrm{HCl}(\mathrm{pH}$ 7.4), $10 \mathrm{mM} \mathrm{NaCl}, 3 \mathrm{mM} \mathrm{MgCl} 2,1 \% \mathrm{BSA}$ and $0.1 \%$ 497 Tween-20) was added and mixed 5 times followed by centrifugation of nuclei ( $500 \mathrm{~g}$ for 5 $498 \min$ at $4^{\circ} \mathrm{C}$ ). After removing the supernatant carefully, nuclei were resuspended in chilled 499 Nuclei Buffer (10X Genomics), filtered by Flowmi Cell Strainer (40uM) and counted using 500 a Countess II FL Automated Cell Counter. Then the nuclei were immediately used to 501 generate scATAC-seq library.

502 ScATAC-seq library was generated using the Chromium Single Cell ATAC Library \& Gel 503 Bead Kit (10X Genomics) following the manufacturer's protocol. We targeted 3000 nuclei 504 with 12 PCR cycles for sample index PCR. Library was checked by $2 \%$ E-gel 505 (Thermofisher Scientific) and quantified using Qubit (Thermofisher Scientific). 506 Sequencing was performed on Illumina NextSeq500 using NextSeq 500/550 High Output 507 Kit v2.5 (Illumina).

508 Raw sequencing reads of the SNU601 ScATAC-seq sample was de-multiplexed with the 509 10x Genomics Cell Ranger ATAC Software (v.1.2.0; https://support.10xgenomics.com/single510 cell-atac/software/pipelines/latest/algorithms/overview) and aligned to the human GRCh38 511 reference genome. The aligned ScATAC-seq data of the two pre-treatment basal cell 512 carcinoma samples (SU006 and SU008) were downloaded from the Gene Expression 513 Omnibus under accession GSE12978523. To obtain all potential SNPs for the SU006 and 514 SU008 samples, GATK Mutect2 was used to call all single-nucleotide variants (SNVs) on 515 the deduplicated bam files by the Picard toolkits of both the t-cell dataset and the tumor 516 dataset from the same tumor. All SNVs from the paired tumor-normal datasets were 
517 combined and the read counts of these SNPs were quantified for each cell in the tumor

518 scATAC-seq dataset. The pre-filtered cell barcodes for the two public scATAC-seq

519 datasets were retrieved from the previous study ${ }^{23}$. For the SNU601 scATAC-seq data, we

520 instead quantified the read counts of the two alleles of the SNPs more reliably called from

521 the paired normal scDNA-seq data. Like scDNA-seq, we applied VarTrix to generate two

522 SNP-by-cell matrices for both reference alleles and alternative alleles of all the SNVs for

523 all the scATAC-seq datasets. To obtain a SNP set including only SNVs that are more

524 possible to be germline SNPs, we further filtered out the SNVs $<20$ reads for the SU008

525 sample and $<30$ reads for the SU006 sample. SNPs with extreme VAF values $<0.1$ or

$526>0.9$ were also excluded for both samples. Since we used the phasing information from

527 the paired scDNA-seq data to assist the estimation of the haplotype structures for the 528 SNU601 ScATAC-seq data, we instead filtered out the cells $<5$ reads and the SNPs $<5$

529 reads to improve quality of the downstream analysis.

\section{$530 \quad$ Linked-reads sequencing and data processing}

531 The three samples with the linked-reads sequencing data were acquired as surgical

532 resections following informed consent under an approved institutional review board

533 protocol from Stanford University. Samples were subjected to mechanical and enzymatic

534 dissociation as previously described, followed by cryopreservation of dissociated cells ${ }^{33}$.

535 Cryofrozen cells were rapidly thawed in a bead bath at $37^{\circ} \mathrm{C}$. Cell counts were obtained

536 on a BioRad TC20 cell counter (Biorad, Hercules, CA) using 1:1 trypan blue dilution.

537 Between 1.5-2.5 million total cells were washed twice in PBS. Centrifugation was carried 538 out at $400 \mathrm{~g}$ for 5 minutes. PBS was removed and cell pellets were frozen at $-80^{\circ} \mathrm{C}$. DNA 
539 extraction was carried out on cell pellets following thawing using either MagAttract HMW

540 DNA Kit (P5931) or AllPrep DNA/RNA Mini Kit (Qiagen Inc., Germantown, MD, USA) as

541 per manufacturer's protocol. Quantification was carried out using Qubit (Thermofisher

542 Scientific).

543 Sequencing libraries were prepared from DNA using Chromium Genome Reagent Kit (v2

544 Chemistry) (10X Genomics, Pleasanton, CA, USA) as per manufacturer's instructions.

545 Sequencing was performed using Illumina HiSeq or NovaSeq sequencers using 150x150

546 bp paired end sequencing and i7 index read of 8 bp. Long Ranger (10X Genomics)

547 version 2.2.0 was used to perform read alignment to GRCh38, calling and phasing of

548 SNPs, indels and structural variants.

\section{Segmentation}

550 The first step of Alleloscope is to segment the genome into regions with different CNA

551 profiles. The appropriate segmentation algorithm depends on what samples are available.

552 First, matched bulk DNA sequencing data (WGS/WES) or pseudo-bulk data from

553 scDNAseq data can be segmented using FACLON ${ }^{5}$, a segmentation method that jointly

554 models the bulk coverage and bulk VAF profiles, if a matched normal sample is available.

555 To accommodate segments from rare subclones, methods that integrate shared cellular

556 breakpoints in CNA detection for scDNA-seq data such as $\mathrm{SCOPE}^{47}$ can improve

557 sensitivity. Since FALCON requires a matched normal sample or a sufficiently large set

558 of normal cells, if these are not available then Alleloscope instead relies on an HMM-

559 based segmentation method. The HMM method, which operates on the binned counts of 560 pooled cells, assumes a Markov transition matrix on four hidden states representing 
561 deletion, copy-neutral state, single-copy amplification and double-copy amplification:

$562\left(\begin{array}{cccc}1-3 t & t & t & t \\ t & 1-3 t & t & t \\ t & t & 1-3 t & t \\ t & t & t & 1-3 t\end{array}\right)$, where $t=1 \times 10^{-6}$ as default. Emission probabilities

563 follow a normal distribution with means equal to $\{1.8,1.2,1,0.5\}$ and standard deviations

564 equals to 0.2. All the scDNA-seq samples were segmented using the HMM algorithm.

565 With the paired sample, the P6198 tumor sample was segmented using FALCON on the

$5661,399,650$ SNPs $>30$ reads across 2,271 cells with all the default parameters.

567 Whole-exome sequencing (WES) data processing

568 The WES data of the two paired tumor-normal samples (SU006 and SU008) were

569 obtained from the Sequence Read Archive under accession PRJNA533341. Raw fastq

570 files were aligned to the GRCh37 reference genome using bwa-mem ${ }^{48}$ with duplicate

571 reads removed using the Picard toolkits ${ }^{49}$. The copy number calls of paired normal-tumor

572 samples were obtained using Varscan $2^{50}$. To perform allele-specific copy number

573 analysis on the WES using FALCON, GATK HaplotypeCaller ${ }^{49}$ was used to call SNPs on

574 both tumor and normal samples. Then FALCON was used to segment each chromosome

575 based on the read counts of the reference alleles and alternative alleles of the SNPs

576 overlapped between the paired tumor-normal samples.

577 SNP Phasing and Single-cell Allele Profile Estimation

578 For each region after segmentation, an expectation-maximization (EM)- based method is 579 used to iteratively phase each SNP and estimate cell-specific allele-specific copy number 580 states for all scDNA-seq and scATAC-seq data sets. Recall that by "major haplotype" we 
581 refer to the haplotype with higher aggregate copy number in the sample. Let $I_{j}$ indicate 582 whether the reference allele of SNP $\mathrm{j}$ is located on the major haplotype and $\theta_{i}$ denote 583 major haplotype proportion of cell i. The EM model iterates the expectation step and the 584 maximization step. The complete log likelihood of the model is

$$
l(\theta)=\sum_{j=1}^{n} \log P\left(A_{i j}, B_{i j} \mid \theta\right)
$$

$$
=\sum_{j=1}^{n}\left\{\left[A_{i j} \log \theta+B_{i j} \log (1-\theta)\right] I_{j}+\left[B_{i j} \log \theta+A_{i j} \log (1-\theta)\right]\left(1-I_{j}\right)\right\}
$$

where $A_{i j}$ and $B_{i j}$ are the observed read counts for the reference and alternative alleles of cell $\mathrm{i}$ on SNP $\mathrm{j}$. In the E-step, we first calculate the expected value of the posterior probability of the hidden variable $I_{j}$ to construct a lower bound for optimization

$$
E_{\widehat{\theta}^{(t)}}\left[I_{j} \mid A_{i j}, B_{i j}\right]=\hat{I}_{j}^{(t)}=\frac{\prod_{i} \hat{\theta}_{i}^{(t)} A^{A_{i j}}\left(1-\hat{\theta}_{i}^{(t)}\right)^{B_{i j}}}{\prod_{i} \hat{\theta}_{i}^{(t)^{A_{i j}}}\left(1-\hat{\theta}_{i}^{(t)}\right)^{B_{i j}}+\prod_{i}\left(1-\hat{\theta}_{i}^{(t)}\right)^{A_{i j}} \hat{\theta}_{i}^{(t)^{B_{i j}}}}
$$

591 where $\hat{\theta}_{i}^{(t)}$ is the parameter from the $\mathrm{t}^{\text {th }}$ iteration. In the M-step, $\hat{\theta}_{i}$ is updated by solving

$$
\begin{gathered}
\hat{\theta}_{i}^{(t+1)}=\operatorname{argmax}_{\theta_{i}} E\left[l(\theta) \mid A_{i j}, B_{i j}, \hat{\theta}_{i}^{(t)}\right] \\
=\frac{\sum_{j}\left[A_{i j} \hat{I}_{j}^{(t)}+B_{i j}\left(1-\hat{I}_{j}^{(t)}\right)\right]}{\sum_{j}\left[A_{i j} \hat{I}_{j}^{(t)}+B_{i j}\left(1-\hat{I}_{j}^{(t)}\right)\right]+\sum_{j}\left[A_{i j}\left(1-\hat{I}_{j}^{(t)}\right)+B_{i j} \hat{I}_{j}^{(t)}\right]}
\end{gathered}
$$

594 Where $\hat{\theta}_{i}^{(t)}$ and $\hat{\theta}_{i}^{(t+1)}$ are from two successive iterations of EM. The two steps are 595 iterated until converge. To speed up the EM process, we limited the maximum number of 
596 SNPs in a region to be 30,000 in our analysis. For the SNU601 scATAC-seq dataset, 597 since the phase were estimated in the paired scDNA-seq dataset with higher depth, we

598 directly applied the estimated $\hat{I}_{j}$ 's from scDNA-seq data to estimate the $\hat{\theta}_{i}$ 's of the cells in 599 the scATAC-seq data. To improve the estimation results, cells with $<20$ read counts 600 covering the identified SNPs were excluded for each region.

601 Selecting normal cells and normal regions for single-cell Coverage Normalization

602 Let $r$ represent a region in the genome after segmentation. To compute the relative 603 coverage change for each cell in region $r\left(\hat{\rho}_{\text {ir }}\right)$, normal cells and diploid regions identified 604 within the sample are required for normalization. After major haplotype proportions for 605 each cell in each region $\hat{\theta}_{i r}$ 's are inferred from the EM-based algorithm, the estimates are 606 used to identify normal cells and diploid regions under a hierarchical clustering of all cells. 607 To identify normal cells, the dendrogram tree is first cut into k largest groups (we used $608 k=5$ which worked well across samples). The cluster with normal cells is identified by 609 selecting the $\mathrm{c}^{\text {th }}$ cluster with the minimum distance calculated by

$$
\sum_{r=1}^{R}\left|\frac{\sum_{\widehat{\theta}_{i} \in S_{c}} \hat{\theta}_{i r}}{n_{c}}-0.5\right|^{2}
$$

611 where $S_{c}$ represents $\hat{\theta}_{i}$ values of the cells in the $c^{\text {th }}$ cluster, and $n_{c}$ is total cell number in 612 the $\mathrm{c}^{\text {th }}$ cluster. All cells in the $\mathrm{c}^{\text {th }}$ cluster are considered as candidate normal cells.

613 Putative diploid regions are next identified in each cluster. Similar to normal cell 614 identification, Alleloscope computes the first measurement $\left(d_{c r}\right)$ as the sum $\hat{\theta}_{i}$ distance 615 of the cells in the $c^{\text {th }}$ cluster for each region $r$ 


$$
d_{c r}=\sum_{\hat{\theta}_{i} \in S_{c}}\left|\hat{\theta}_{i r}-0.5\right|^{2}
$$

617 Since amplified regions with both haplotypes equally amplified can also have small sum

$618 \hat{\theta}_{i}$ distance, adjusted raw coverages are also considered in diploid region selection. The

619 adjusted raw coverage of cell $\mathrm{i}$ in region $\mathrm{r}\left(\tilde{\rho}_{i R}\right)$ is computed by

$$
\tilde{\rho}_{i r}=\frac{N_{i r}}{N_{i}} \times \frac{L_{r}}{L L},
$$

621 where $N_{i r}$ is the total read counts in region r of cell $\mathrm{i}$ and $N_{i}$ is the total read counts of cell

622 i across the regions. $L_{r}$ is length of the region $\mathrm{r}$ and $L L$ is total length of the genome. For

623 region $\mathrm{r}$, cells with $\tilde{\rho}_{i r}$ values larger than the $99^{\text {th }}$ percentile are assigned the $\tilde{\rho}_{i r}$ values

624 equal to the $99^{\text {th }}$ percentile across the cells. The second measurement $\left(m_{c r}\right)$ used to 625 select diploid regions in the $\mathrm{c}^{\text {th }}$ cluster is the mean $\tilde{\rho}_{\text {ir }}$ for each region $\mathrm{r}$

$$
m_{c r}=\frac{\sum_{\widetilde{\rho}_{i} \in S_{c}} \tilde{\rho}_{i r}}{n_{c}}
$$

627 where $S_{c}$ here represents $\tilde{\rho}_{i}$ values of the cells in the $c^{\text {th }}$ cluster. To identify diploid regions, $628 d_{c r}$ and $m_{c r}$ are both ranked from the smallest to the largest for each cluster c.

629 Alleloscope shows a list of potential diploid regions for each cluster by raking the sums 630 of $d_{c r}$ ranks and $m_{c r}$ ranks. Excluding the $\mathrm{c}^{\text {th }}$ cluster identified as the normal group,

631 Alleloscope proposed a list for the candidate diploid regions across the clusters by 632 selecting the majority region.

633 Since coverage on scATAC-seq data is confounded by the epigenetic signals, 634 chromosome 22 for SU008 and chromosome 18 for SU006 were directly selected as 
635 normal regions based on the WES data. Individual cells were classified into normal and

636 tumor cells based on the epigenetic signals on the scATAC-seq data. For the SNU601

637 SCATAC-seq dataset, chromosome 10 was selected as the normal region based on the 638 paired scDNA-seq data.

\section{Cell-level Genotyping}

640 The cell-level allele-specific copy number profiles are defined by both relative coverage 641 change $\left(\hat{\rho}_{i r}\right)$ and major haplotype proportion $\left(\hat{\theta}_{i r}\right)$ of region $\mathrm{r}$ and cell i. After the normal 642 cells and normal control region are identified, cell-specific relative coverage change in 643 region $r$ is calculated as

$$
\hat{\rho}_{i r}=\frac{N_{i r}}{N_{i 0}} / \operatorname{median}\left(\frac{N_{0 r}}{N_{00}}\right)
$$

645 where $N_{i r}$ is total read counts in region $r$ and $N_{i 0}$ is total read counts in a reference region 646 of cell i. $N_{0 r}$ is a vector denoting total read counts in region $\mathrm{r}$ of all identified normal cells 647 and $N_{00}$ is a vector denoting total read counts in the same reference region $r$ of all 648 identified normal cells. Since SNU601 is a tumor cell line with no normal cells in the 649 dataset, $N_{0 r}$ and $N_{00}$ were calculated from the cells in the matched normal P6198 sample 650 as a substitute for the scDNA-seq data. For SNU601 scATAC-seq data, we aligned the

651 distribution of the $\hat{\rho}_{i r}$ values in paired scDNA-seq data to the distribution of the $\frac{N_{i r}}{N_{i}}$ values

652 for each region to get the normalized $\hat{\rho}_{i r}$ in the scATAC-seq data. The normalized $\hat{\rho}_{i r}$ 653 values for the scATAC-seq data were computed by

$$
\hat{\rho}_{i r}^{a t a c}=\frac{N_{i r}}{N_{i 0}} / \operatorname{median}\left(\frac{N_{i r}}{N_{i 0}}\right) \times \operatorname{median}\left(\hat{\rho}_{i r}^{\text {dna }}\right)
$$


655 Next, cells with extreme $\hat{\rho}_{i r}$ values larger than the $99^{\text {th }}$ percentile and smaller than the

656 first percentile across the cells are considered outliers and excluded for each region. With

657 the $\left(\hat{\rho}_{i r}, \hat{\theta}_{i r}\right)$ pairs, cells in the scDNA-seq data can be classified into the haplotype profiles

658 (g) with the expected $\left(\rho_{g}, \theta_{g}\right)$ values based on minimum distance. Although signals in the 659 scATAC-seq data are much noisier, the haplotype structures identified in the paired 660 scDNA-seq data can help to guide the genotyping for each region. In region $r$, the 661 posterior probability of cell $\mathrm{i}$ carrying a haplotype profile observed in region $r$ in the paired 662 scDNA-seq data was

$$
P\left(G T_{i r}=g_{r} \mid \hat{\rho}_{i r}, \hat{\theta}_{i r}\right)=\frac{P\left(\hat{\rho}_{i r}, \hat{\theta}_{i r} \mid G T_{i r}=g_{r}\right) \pi_{g_{r}}}{\sum_{g_{r^{\prime}}} P\left(\hat{\rho}_{i r}, \hat{\theta}_{i r} \mid G T_{i r}=g^{\prime}\right) \pi_{g_{r}^{\prime}}}
$$

664 where $g_{r}$ denotes the haplotype profiles observed in region $r$ in the paired scDNA-seq 665 data and $\pi_{g_{r}}$ denotes the prior probability that a randomly sampled cell carrying the $g_{r}$ 666 haplotype profile. A uniform prior can be used for $\pi_{g_{r}}$ in the absence of external 667 information. In the formula,

$$
P\left(\hat{\rho}_{i r}, \hat{\theta}_{i r} \mid G T_{i r}=g_{r}\right)=P\left(\hat{\rho}_{i r} \mid \mu=\rho_{g} ; \sigma_{\rho}=0.25\right) \times P\left(\hat{\theta}_{i r} \mid \mu=\theta_{g} ; \sigma=\sqrt{\frac{\hat{\theta}_{i r}\left(1-\hat{\theta}_{i r}\right)}{n_{i r}}}\right),
$$

669 where $n_{i r}$ is the number of total read counts in region $r$ for cell $i$. The haplotype profile of 670 cell $\mathrm{i}$ in region $\mathrm{r}$ was estimated by maximizing the above posterior probability. The 671 haplotype profiles of each region are visualized using different colors in the two672 dimensional scatter plots for both scDNA-seq and scATAC-seq data with the confidence 
673 scores calculated using the distance of the points to the canonical centers and the

674 standard deviations.

675 Validations using paired linked-reads sequencing data

676 We validated our algorithm using paired linked-reads sequencing data with two strategies

677 in one gastric cancer patient sample and two colorectal cancer patient samples. First, the

678 phasing accuracy was assessed by comparing the estimated SNP phases on the scDNA-

679 seq data and the known phases of the same SNPs from the linked-reads sequencing data

680 in individual regions. In the linked-reads sequencing data, SNPs within the same phase

681 sets are phased with respect to one another, while those between different SNP sets are

682 not. Therefore, we compared the phases of the SNPs overlapping between our estimated

683 SNP set and the phase set with the largest numbers of SNPs in the linked-reads

684 sequencing data for each region. The reference alleles of the overlapping SNPs with $\hat{I}_{j}$

$685>0.5$ are estimated to be on the major haplotype. Otherwise, the reference alleles of the

686 overlapping SNPs with $\hat{I}_{j}<0.5$ are estimated to be on the minor haplotype. The SNPs with

$687 \hat{I}_{j}=0.5$ are excluded. By comparing estimated phases and known phases from the linked-

688 reads sequencing data of the overlapping SNPs, the phasing accuracy was computed for

689 each region.

690 Secondly, we evaluated the genotyping accuracy by comparing the estimated haplotype

691 profiles of each cell and the haplotype profiles inferred from the linked-reads sequencing

692 data in individual regions. In the linked-reads sequencing data, the phase set with the

693 largest numbers of SNPs was selected. The known phases of the overlapping SNPs

694 between the phase set and the estimated SNPs were used to infer $\theta_{i}$ for each cell. Cell- 
695 level haplotype profiles using $\theta_{i}$ 's from linked-reads sequencing data were considered as 696 gold standard. By comparing the estimated haplotype profiles from $\hat{\theta}_{i}$ and the haplotype

697 profiles from $\theta_{i}$, genotyping accuracy was computed for each region. If the number of 698 overlapping SNPs for an amplified region is smaller than 5000 , the phase sets were 699 combined from the largest to the smallest to reduce variance of $\theta_{i}$ 's inferred from linked700 reads sequencing data. The estimated SNP phases $\left(\hat{I}_{j}\right)$ were used as templates to 701 combine separate phase sets.

\section{Cell Lineage Reconstruction}

703 To investigate the tumor subclonal structure for scDNA-seq data, cell-specific haplotype 704 profiles from Alleloscope across the genome were used to reconstruct cell lineage trees.

705 The "Gower's distance" is calculated using "cluster" R package on the nominal haplotype 706 profiles between cells. Then hierarchical clustering is performed on the distance using the

707 "ward.D2" method. Since variance of $\hat{\theta}_{i}$ 's is higher when fewer SNPs are located in a 708 segment, we included the segments with more than 2,000 SNPs identified. The clustering 709 results are visualized using the 'pheatmap' R package. Each segment was plotted with 710 its length proportional to $5,000,000$ bins. The heights of the clustering tree were log711 transformed for easier visualization.

712 The tumor subclonal structures were also investigated in the scATAC-seq data. Instead

713 of using the haplotype profiles defined by the $\left(\hat{\rho}_{i r}, \hat{\theta}_{i r}\right)$ pairs, the cells from the two public 714 basal cell carcinoma were clustered using $\hat{\theta}_{i r}$ values, which are orthogonal to the peak 715 signals based on total coverage, across the segments with more than 500 SNPs. Then 716 hierarchical clustering is performed on the Euclidean distance using the "ward.D2" 
717 method and visualized using 'pheatmap' $\mathrm{R}$ package with the three largest clusters 718 separated by marginal lines. The heights of the clustering tree were log-transformed for 719 easier visualization.

720 Since the subclones for the SNU601 sample were identified first from the scDNA-seq data,

721 for this cell we adopted a supervised strategy to assign each cell into different subclones.

722 First, we identified 10 marker regions-- chr1b, 3b-d, 4b, 7a, 11b, 13b, and 20b-c that help

723 to differentiate the cells into the six major subclones based on the subclone specific copy

724 number profiles from the scDNA-seq data. Combining the haplotype profiles across the 725 ten regions for each cell enables assignment of the cells into one of the six subclones 726 with high confidence. The posterior probability of cell i coming from clone k was

$$
P\left(\text { Clone }_{i}=k \mid \hat{\rho}_{i}, \hat{\theta}_{i}\right)=\frac{P\left(\hat{\rho}_{i}, \hat{\theta}_{i} \mid \text { Clone }_{i}=k\right) \pi_{k}}{\sum_{k^{\prime}} P\left(\hat{\rho}_{i}, \hat{\theta}_{i} \mid \text { Clone }_{i}=k^{\prime}\right) \pi_{k^{\prime}}},
$$

728 where $k \in\{1 \sim 6\}$ for the six clones and $\pi_{k}$ is the prior probability that a randomly sampled

729 cell coming from the $k^{\text {th }}$ clone, which can be estimated from the paired scDNA-seq data

730 or set to uniform (in our analysis setting to uniform gives very similar results). In the 731 formula,

$$
\begin{aligned}
P\left(\hat{\rho}_{i}, \hat{\theta}_{i} \mid \text { Clone }_{i}\right. & =k)=\prod_{x} P\left(\hat{\theta}_{i x}, \hat{\rho}_{i x} \mid \text { Clone }_{i}=k\right) \\
& =\prod_{x} \phi\left(\frac{\hat{\theta}_{i x}-\theta_{k x}}{\sqrt{n_{i x} \theta_{k x}\left(1-\theta_{k x}\right)}}\right) \phi\left(\frac{\hat{\rho}_{i x}-\rho_{k x}}{\sigma_{\rho}}\right),
\end{aligned}
$$

734 where $x$ is the index for the ten marker regions, $\hat{\theta}_{i x}$ and $\hat{\rho}_{i x}$ are the estimated major 735 haplotype proportion and relative coverage for cell $\mathrm{i}$ in the scATAC-seq data, $\theta_{k x}$ and $\rho_{k x}$ 
736 are the "known values" for specific haplotype profiles for clone k derived from the paired

737 scDNA-seq data, and $n_{i x}$ is the number of total read counts in the $x^{\text {th }}$ marker region for

738 cell i. Each cell was assigned into one of the six subclones by maximizing the above

739 posterior probability with the confidence score being the posterior probability of the

740 assigned clone.

741 ScATAC-seq data analysis

742 To investigate the relationships between allele-specific CNAsand chromatin accessibility,

743 for each cell in SCATAC-seq data we processed the peak signals in addition to the allele-

744 specific CNAs. For the two public basal cell carcinoma samples, the peak by cell matrices

745 was obtained from GSE129785. We subset the fragment counts for each peak in the cells

746 from the SU008 sample, regressed out cell total coverage for each peak by linear

747 regression, and projected the cells onto the UMAP plot using genome-wide peak signals ${ }^{51}$.

748 The cell type identify for each cluster was retrieved from the labels in the previous study ${ }^{23}$.

749 To further explore intratumor heterogeneity, we selected the cells labeled as tumor cells,

750 regressed out cell total coverage, and projected the tumor cells onto the UMAP plot like

751 previously described. Then the DNA level information and epigenetic signals for each cell

752 can be visualize and analyzed together.

753 For the SNU601 scATAC-seq dataset, scATAC-pro ${ }^{52}$ was used to call peaks and 754 generate the peak by cell matrix from the bam file and fragment file output by the Cell 755 Ranger software. We first filtered out the cells that have proportions of fragments on the 756 detected peaks $<0.4$ and or total peaks outside of the range $15,000 \sim 100,000$, and filtered

757 out the peaks observed in less than 0.1 of cells. Next, we regressed out cell total coverage 
758 for each peak by linear regression, and projected the cells onto the UMAP plot using

759 genome-wide peak signals. Then the clonal assignment based on the DNA information

760 and the peak signals can be integrated at the single-cell level.

761 Based on the lineage structure from the paired scDNA-seq data, the cells can also be

762 placed in the lineage tree based on their clonal assignment. Under the lineage structure,

763 pairwise comparison using Chi-squared test was performed on the proportion of the to 764 identify differential accessible peaks (DAPs) for each branch. A peak was considered a

765 DAP if the FDR adjusted $p$-values $<0.05$. Since copy number alterations are confounding 766 factors that also affect the peak signals, the DAPs were further divided into two groups-

767 1. "CNA" group if the DAPs are in the CNA regions and both signals are positive correlated;

768 2. "Other" group if the DAPs are not categorized in the first group. A set of DAPs were 769 considered to be enriched in the CNA regions if the p-values $<0.05$ under the

770 hypergeometric test. This type of analysis enables investigation of the relationships

771 between the two signals. Each DAP was further mapped to the genes that are potentially

772 regulated based on the $\pm 2,000$ bp distance on the genome. To further visualize the

773 difference of the peak signals among the six clones, the peak signals were pooled across

774 the cells and normalized by the total cell number in each subclone.

\section{$775 \quad$ Data availability}

776 All the linked-reads sequencing data and the scATAC-seq dataset are available under

777 accession \#\#\#. There are no restrictions on data availability or use. The patient scDNA778 seq data were obtained from dbGAP under accession phs001818.v3.p133 (all except 7795931 scDNA) and phs001711'12 (5931 scDNA). The cell line scDNA-seq dataset was from 
780 the Sequence Read Archive (SRA) under accession PRJNA498809. The public scATAC-

781 seq data and whole exome sequencing data were obtained from the SRA under

782 accession PRJNA53277423 and PRJNA533341 ${ }^{37}$.

\section{Code availability}

784 Alleloscope is available on GitHub at https://github.com/seasoncloud/Alleloscope.

\section{Reference}

786 1. Baylin, S.B. \& Jones, P.A. A decade of exploring the cancer epigenome - biological 787 and translational implications. Nat Rev Cancer 11, 726-734 (2011).

788 2. Sandoval, J. \& Esteller, M. Cancer epigenomics: beyond genomics. Curr Opin $789 \quad$ Genet Dev 22, 50-55 (2012).

790 3. Greaves, M. \& Maley, C.C. Clonal evolution in cancer. Nature 481, 306-313 (2012).

791 4. Burrell, R.A., McGranahan, N., Bartek, J. \& Swanton, C. The causes and 792 consequences of genetic heterogeneity in cancer evolution. Nature 501, 338-345 $793 \quad$ (2013).

794 5. Chen, H., Bell, J.M., Zavala, N.A., Ji, H.P. \& Zhang, N.R. Allele-specific copy 795 number profiling by next-generation DNA sequencing. Nucleic Acids Res 43, e23 $796 \quad$ (2015).

797 6. Favero, F. et al. Sequenza: allele-specific copy number and mutation profiles from 798 tumor sequencing data. Ann Oncol 26, 64-70 (2015).

799 7. Ha, G. et al. TITAN: inference of copy number architectures in clonal cell 800 populations from tumor whole-genome sequence data. Genome Res 24, 1881$801 \quad 1893(2014)$. 
802 8. Shen, R. \& Seshan, V.E. FACETS: allele-specific copy number and clonal heterogeneity analysis tool for high-throughput DNA sequencing. Nucleic Acids Res 44, e131 (2016).

805 9. Jamal-Hanjani, M. et al. Tracking the Evolution of Non-Small-Cell Lung Cancer. $N$ Engl J Med 376, 2109-2121 (2017).

807 10. Zaccaria, S. \& Raphael, B.J. Characterizing allele- and haplotype-specific copy numbers in single cells with CHISEL. Nat Biotechnol (2020).

809 11. Van Loo, P. et al. Allele-specific copy number analysis of tumors. Proc Natl Acad Sci U S A 107, 16910-16915 (2010).

811 12. Andor, N. et al. Joint single cell DNA-seq and RNA-seq of gastric cancer cell lines reveals rules of in vitro evolution. NAR Genom Bioinform 2, Iqaa016 (2020).

813 13. Bakker, B. et al. Single-cell sequencing reveals karyotype heterogeneity in murine 814 and human malignancies. Genome Biol 17, 115 (2016).

815 14. Garvin, T. et al. Interactive analysis and assessment of single-cell copy-number $816 \quad$ variations. Nat Methods 12, 1058-1060 (2015).

817 15. Kim, C. et al. Chemoresistance Evolution in Triple-Negative Breast Cancer 818 Delineated by Single-Cell Sequencing. Cell 173, 879-893 e813 (2018).

819 16. Laks, E. et al. Clonal Decomposition and DNA Replication States Defined by 820 Scaled Single-Cell Genome Sequencing. Cell 179, 1207-1221 e1222 (2019).

821 17. Navin, N. et al. Tumour evolution inferred by single-cell sequencing. Nature $\mathbf{4 7 2}$, $822 \quad$ 90-94 (2011).

823 18. Velazquez-Villarreal, E.I. et al. Single-cell sequencing of genomic DNA resolves 824 sub-clonal heterogeneity in a melanoma cell line. Commun Bio/ 3, 318 (2020). 
825 19. Wang, Y. et al. Clonal evolution in breast cancer revealed by single nucleus 826 genome sequencing. Nature 512, 155-160 (2014).

827 20. Corces, M.R. et al. The chromatin accessibility landscape of primary human $828 \quad$ cancers. Science 362 (2018).

829 21. Granja, J.M. et al. Single-cell multiomic analysis identifies regulatory programs in 830 mixed-phenotype acute leukemia. Nat Biotechnol 37, 1458-1465 (2019).

831 22. Litzenburger, U.M. et al. Single-cell epigenomic variability reveals functional cancer heterogeneity. Genome Biol 18, 15 (2017).

833 23. Satpathy, A.T. et al. Massively parallel single-cell chromatin landscapes of human 834 immune cell development and intratumoral T cell exhaustion. Nat Biotechnol 37, $835925-936(2019)$.

836 24. Schep, A.N., Wu, B., Buenrostro, J.D. \& Greenleaf, W.J. chromVAR: inferring 837 transcription-factor-associated accessibility from single-cell epigenomic data. Nat $838 \quad$ Methods 14, 975-978 (2017).

839 25. Cabal-Hierro, L. et al. Chromatin accessibility promotes hematopoietic and $840 \quad$ leukemia stem cell activity. Nat Commun 11, 1406 (2020).

841 26. Dravis, C. et al. Epigenetic and Transcriptomic Profiling of Mammary Gland 842 Development and Tumor Models Disclose Regulators of Cell State Plasticity. $843 \quad$ Cancer Cell 34, 466-482 e466 (2018).

844 27. Guilhamon, P. et al. Single-cell chromatin accessibility in glioblastoma delineates 845 cancer stem cell heterogeneity predictive of survival. bioRxiv, 370726 (2020).

846 28. Pan, D. et al. A major chromatin regulator determines resistance of tumor cells to $847 \quad$ T cell-mediated killing. Science 359, 770-775 (2018). 
848 29. Qu, K. et al. Chromatin Accessibility Landscape of Cutaneous T Cell Lymphoma and Dynamic Response to HDAC Inhibitors. Cancer Cell 32, 27-41 e24 (2017).

850 30. Shaffer, S.M. et al. Rare cell variability and drug-induced reprogramming as a 851 mode of cancer drug resistance. Nature 546, 431-435 (2017).

852 31. Shu, S. et al. Synthetic Lethal and Resistance Interactions with BET Bromodomain 853 Inhibitors in Triple-Negative Breast Cancer. Mol Cell 78, 1096-1113 e1098 (2020).

854 32. Tirosh, I. et al. Dissecting the multicellular ecosystem of metastatic melanoma by $855 \quad$ single-cell RNA-seq. Science 352, 189-196 (2016).

856 33. Sathe, A. et al. The cellular genomic diversity, regulatory states and networking of 857 the metastatic colorectal cancer microenvironment. bioRxiv (2020).

858 34. Bell, J.M. et al. Chromosome-scale mega-haplotypes enable digital karyotyping of 859 cancer aneuploidy. Nucleic Acids Res 45, e162 (2017).

860 35. Greer, S.U. et al. Linked read sequencing resolves complex genomic 861 rearrangements in gastric cancer metastases. Genome Med 9, 57 (2017).

862 36. Zheng, G.X. et al. Haplotyping germline and cancer genomes with high-throughput $863 \quad$ linked-read sequencing. Nat Biotechnol 34, 303-311 (2016).

864 37. Yost, K.E. et al. Clonal replacement of tumor-specific T cells following PD-1 blockade. Nat Med 25, 1251-1259 (2019).

866 38. $\mathrm{Yu}$, J. et al. REC8 functions as a tumor suppressor and is epigenetically 867 downregulated in gastric cancer, especially in EBV-positive subtype. Oncogene 36, 868 182-193 (2017).

869 39. McFarlane, R.J. \& Wakeman, J.A. Meiosis-like Functions in Oncogenesis: A New $870 \quad$ View of Cancer. Cancer Res 77, 5712-5716 (2017). 
871 40. Aqeilan, R.I. et al. Loss of WWOX expression in gastric carcinoma. Clin Cancer $872 \quad$ Res 10, 3053-3058 (2004).

873 41. Baryla, I., Styczen-Binkowska, E. \& Bednarek, A.K. Alteration of WWOX in human $874 \quad$ cancer: a clinical view. Exp Biol Med (Maywood) 240, 305-314 (2015).

875 42. Watkins, T.B.K. et al. Pervasive chromosomal instability and karyotype order in 876 tumour evolution. Nature (2020).

877 43. Gupta, I. et al. Single-cell isoform RNA sequencing characterizes isoforms in $878 \quad$ thousands of cerebellar cells. Nat Biotechnol (2018).

879 44. Lebrigand, K., Magnone, V., Barbry, P. \& Waldmann, R. High throughput error corrected Nanopore single cell transcriptome sequencing. Nat Commun 11, 4025 (2020).

882 45. Singh, M. et al. High-throughput targeted long-read single cell sequencing reveals 883 the clonal and transcriptional landscape of lymphocytes. Nat Commun 10, 3120 884 (2019).

885 46. Zhu, C., Preissl, S. \& Ren, B. Single-cell multimodal omics: the power of many. $886 \quad$ Nat Methods 17, 11-14 (2020).

887 47. Wang, R., Lin, D.Y. \& Jiang, Y. SCOPE: A Normalization and Copy-Number $888 \quad$ Estimation Method for Single-Cell DNA Sequencing. Cell Syst 10, 445-452 e446 889 (2020).

890 48. Li, H. \& Durbin, R. Fast and accurate short read alignment with Burrows-Wheeler 891 transform. Bioinformatics 25, 1754-1760 (2009). 
892 49. McKenna, A. et al. The Genome Analysis Toolkit: a MapReduce framework for analyzing next-generation DNA sequencing data. Genome Res 20, 1297-1303 (2010).

895 50. Koboldt, D.C. et al. VarScan 2: somatic mutation and copy number alteration discovery in cancer by exome sequencing. Genome Res 22, 568-576 (2012).

897 51. Mclnnes, L., Healy, J. \& Melville, J. Umap: Uniform manifold approximation and projection for dimension reduction. arXiv preprint arXiv:1802.03426 (2018).

899 52. Yu, W., Uzun, Y., Zhu, Q., Chen, C. \& Tan, K. scATAC-pro: a comprehensive workbench for single-cell chromatin accessibility sequencing data. Genome Biol 21, 94 (2020).

\section{Acknowledgements}

903 The work is supported by the National Institutes of Health [P01HG00205ESH to B.T.L., 904 S.M.G. AND H.P.J., 5R01-HG006137-07 and 1U2CCA233285-01 to C-Y.W. and to 905 N.R.Z.]. Additional support to HPJ came from the Research Scholar Grant, RSG-13-297906 01-TBG from the American Cancer Society, Clayville Foundation and the Gastric Cancer 907 Foundation.

\section{Author contributions}

909 C.-Y.W. and N.R.Z. conceived the computational methods and designed the study with 910 help from H.P.J. C.-Y.W. developed and implemented the computational methods and

911 conducted all data analyses. B.T.L. helped in data interpretation. B.T.L., H.K. and A.S.

912 performed all related sample preparation and sequencing. S.M.G. performed data pre913 processing and coordinated data transfer. H.P.J. advised all experiments and data 
914 collection. C.-Y.W., N.R.Z, and H.P.J. wrote the manuscript. All authors read and

915 approved the final manuscript.

916 Competing interests

917 The authors declare no competing interests. 

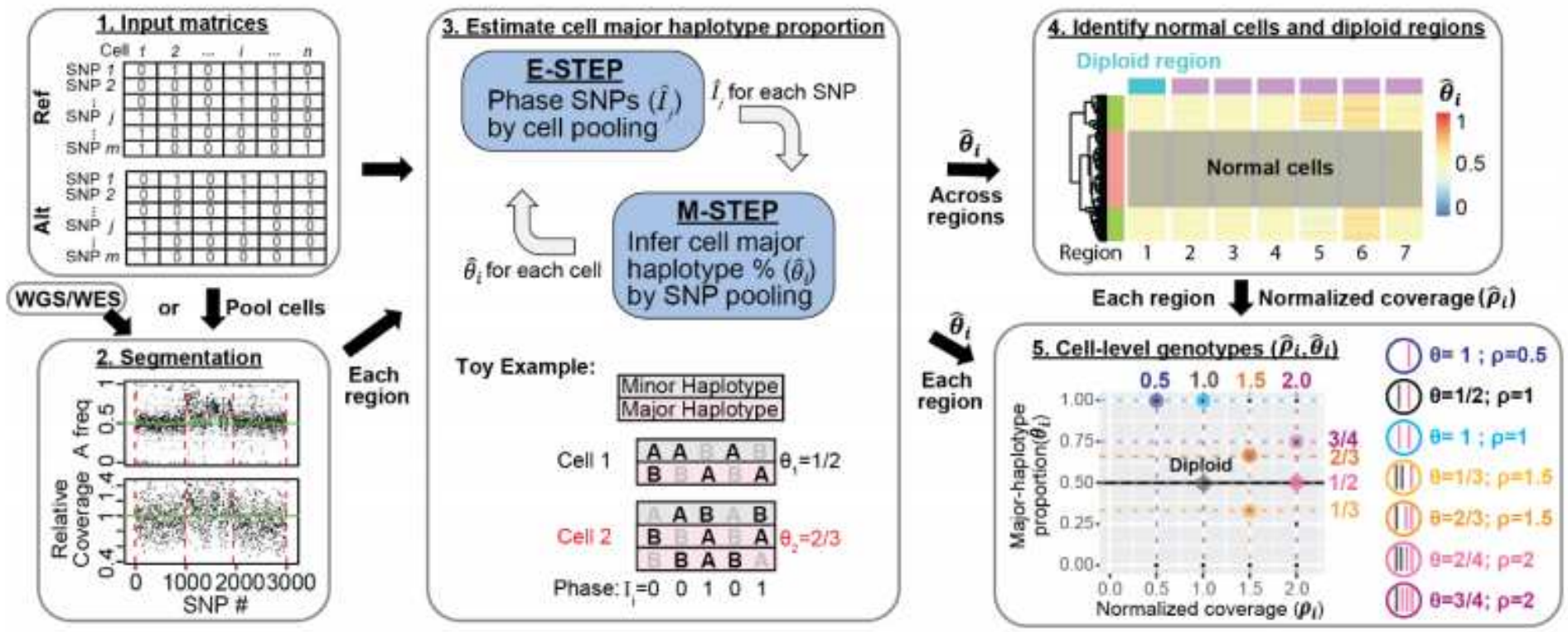

\section{Figure 1}

[Please see the manuscript file to view the figure caption.]

a

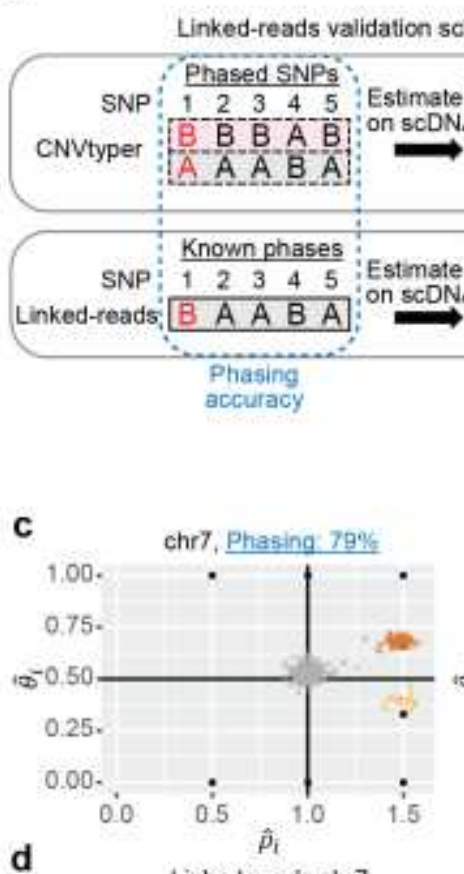

d

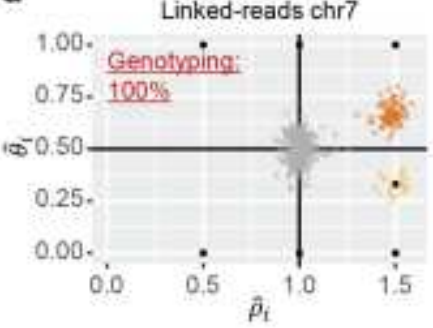

c b

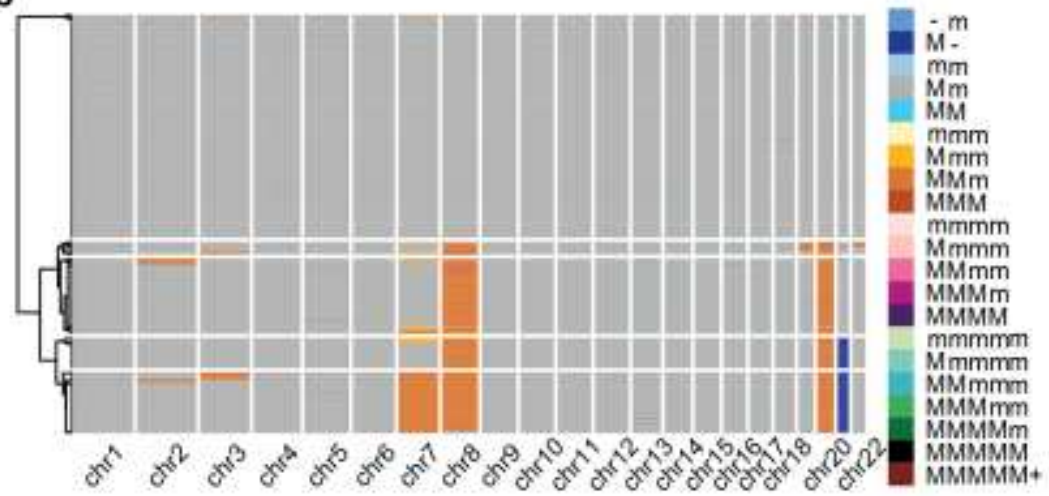

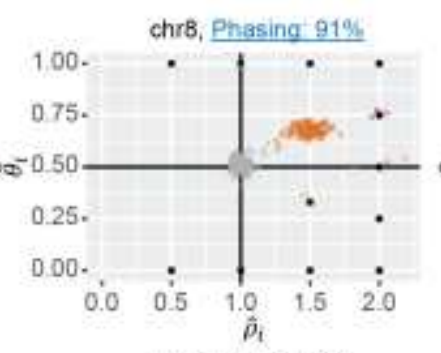
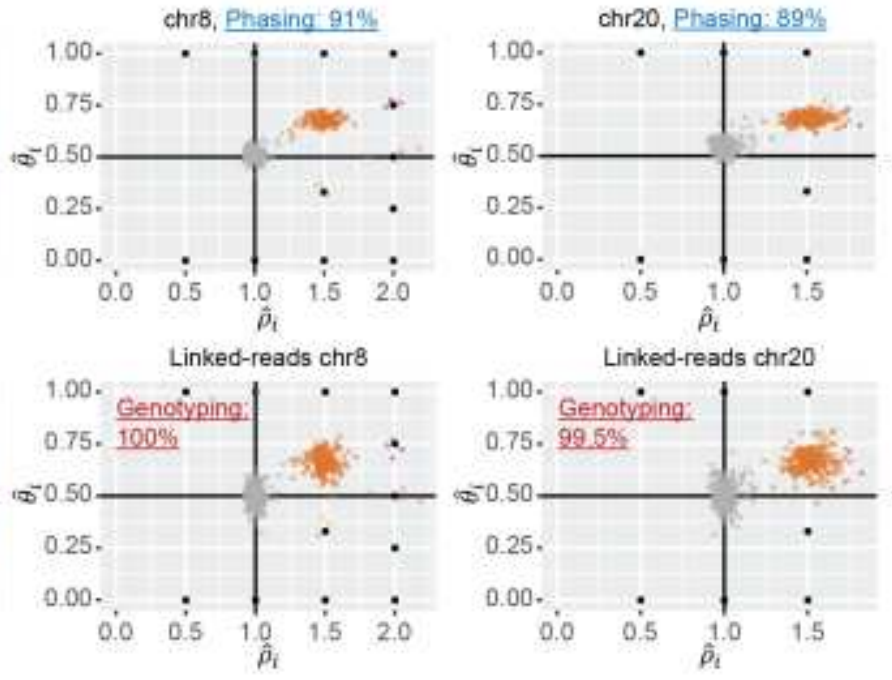
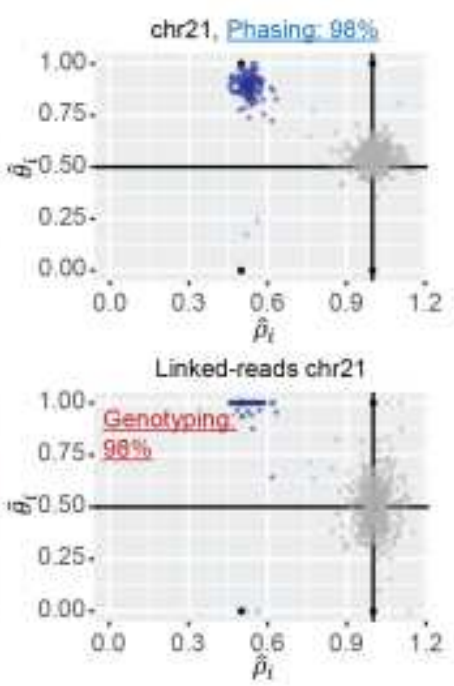
Figure 2

[Please see the manuscript file to view the figure caption.]

a

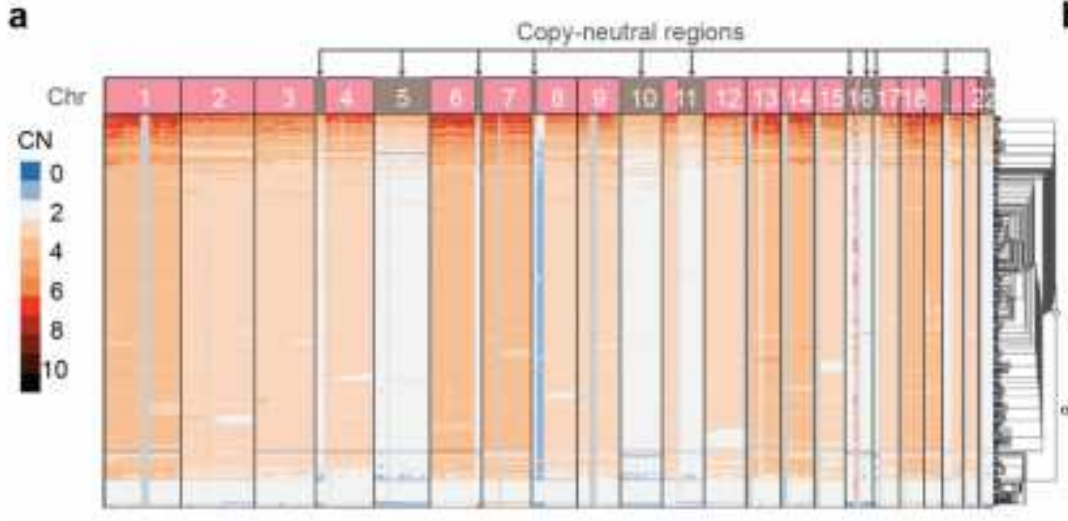

b

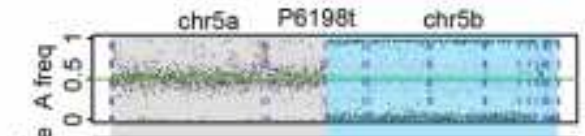

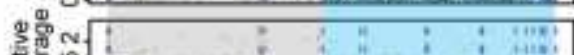

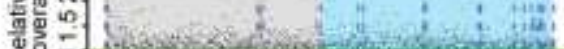

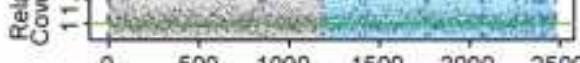

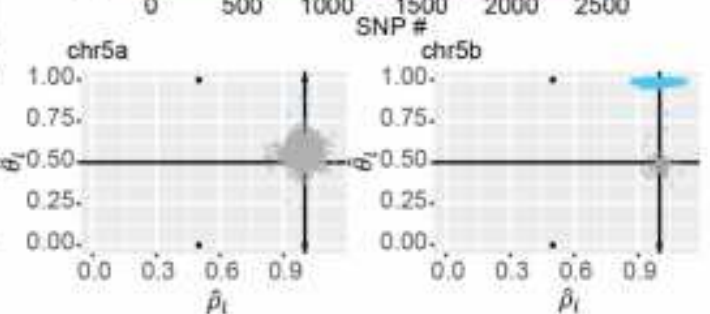

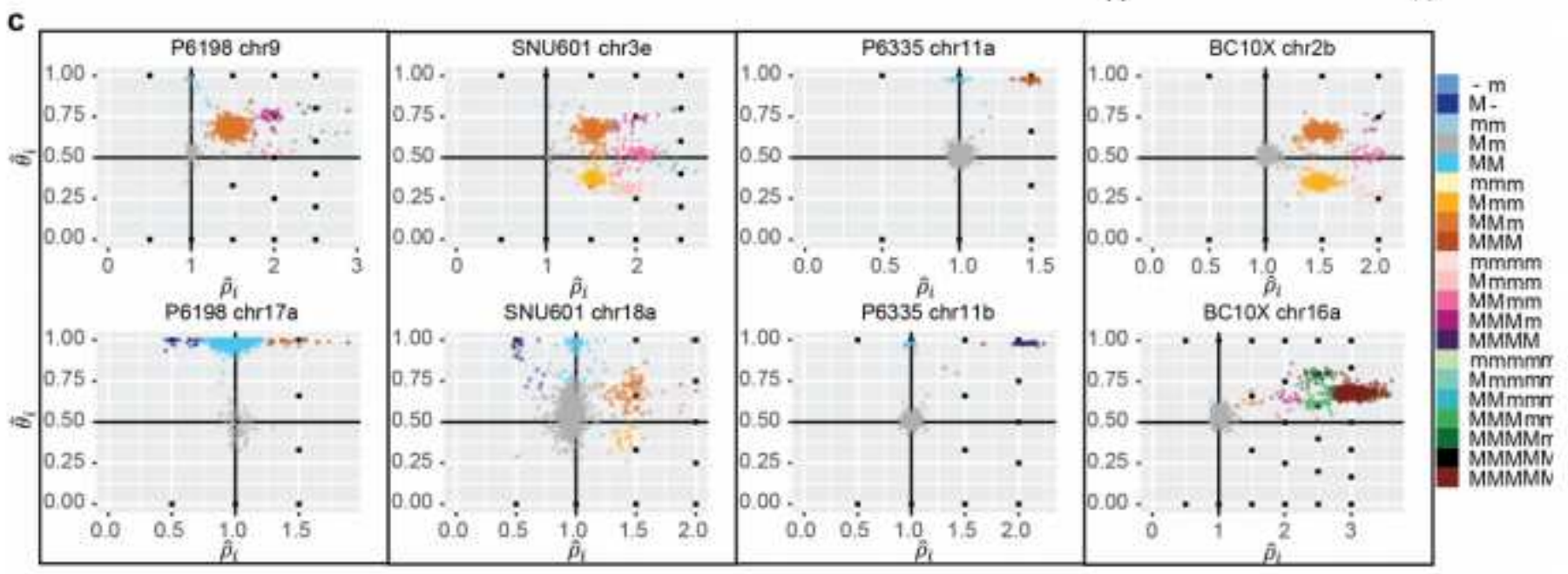

Figure 3

[Please see the manuscript file to view the figure caption.] 

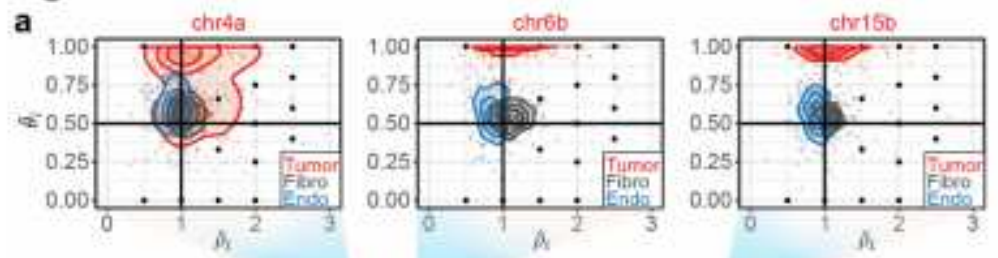

b
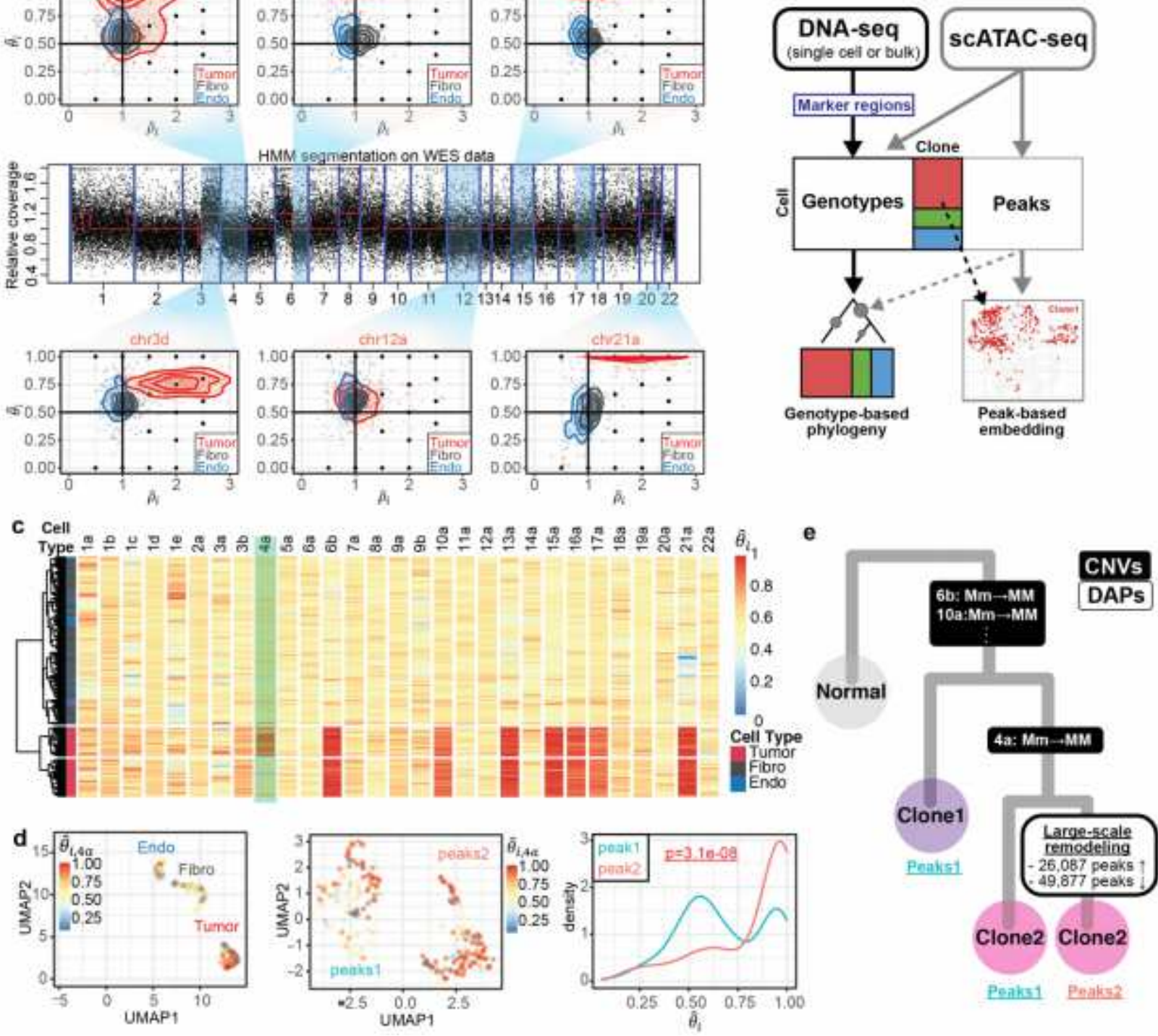

Figure 4

[Please see the manuscript file to view the figure caption.] 
a

HMM segmentation on total coverage

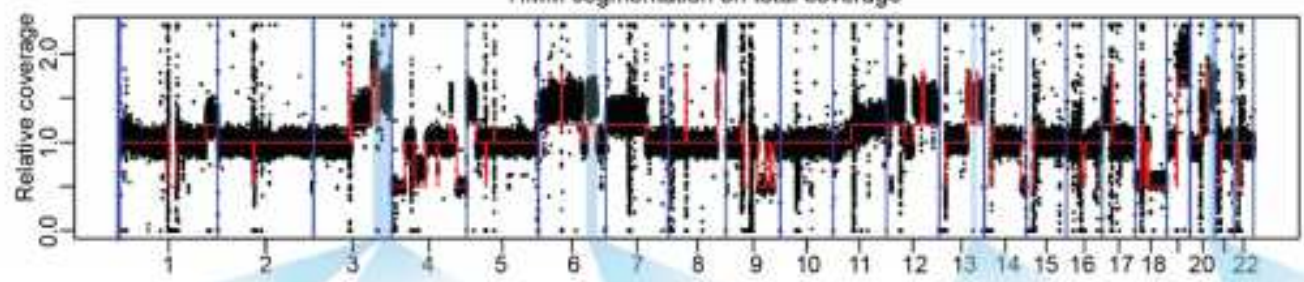

b
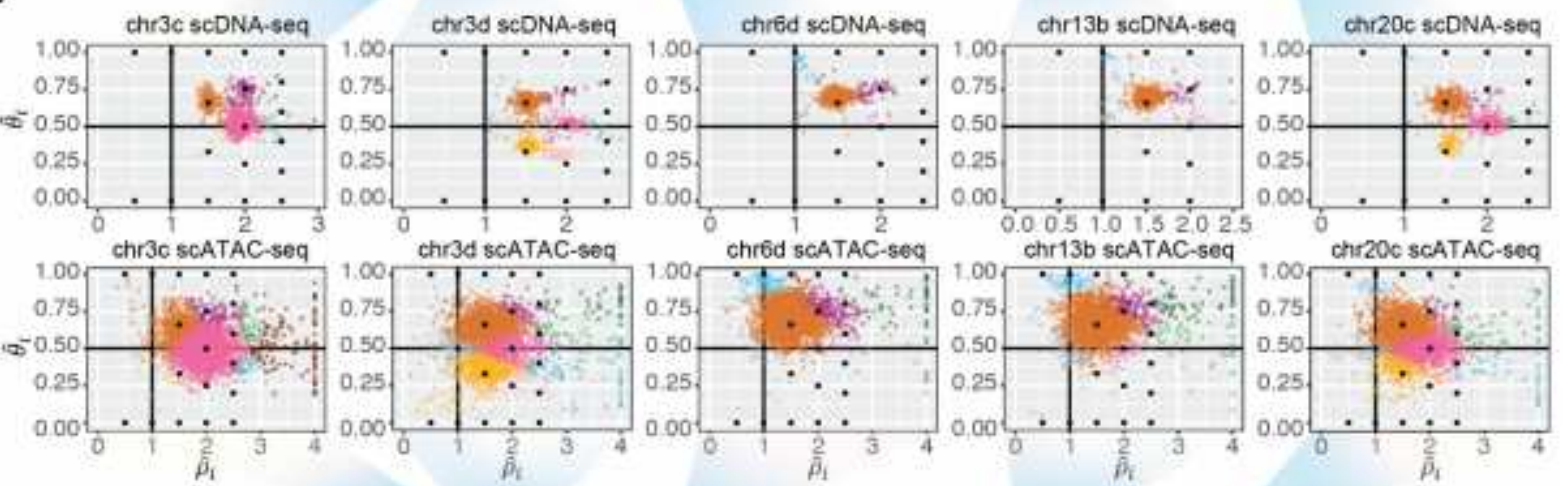

c

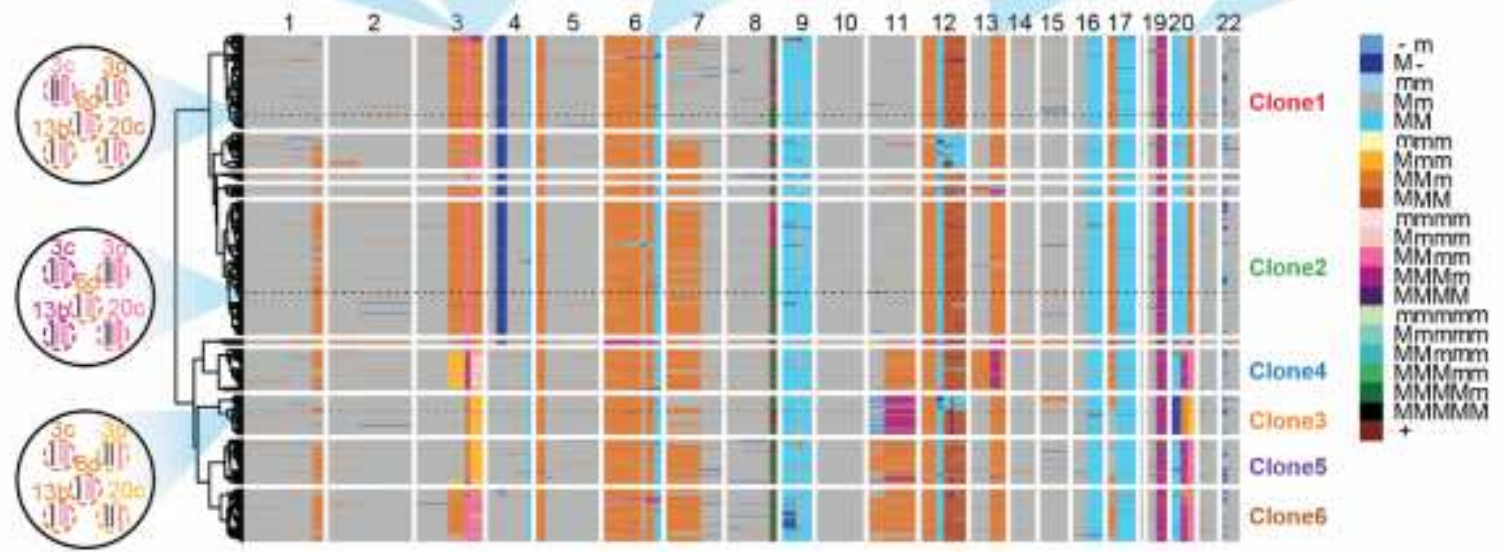

Figure 5

[Please see the manuscript file to view the figure caption.] 
a

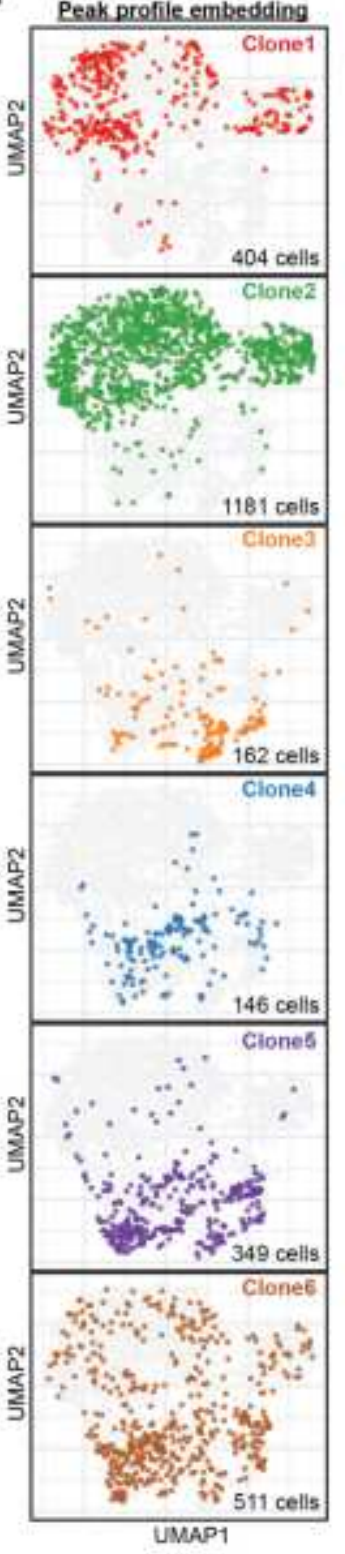

b

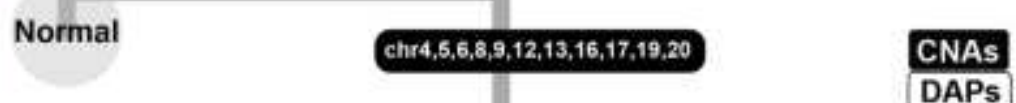

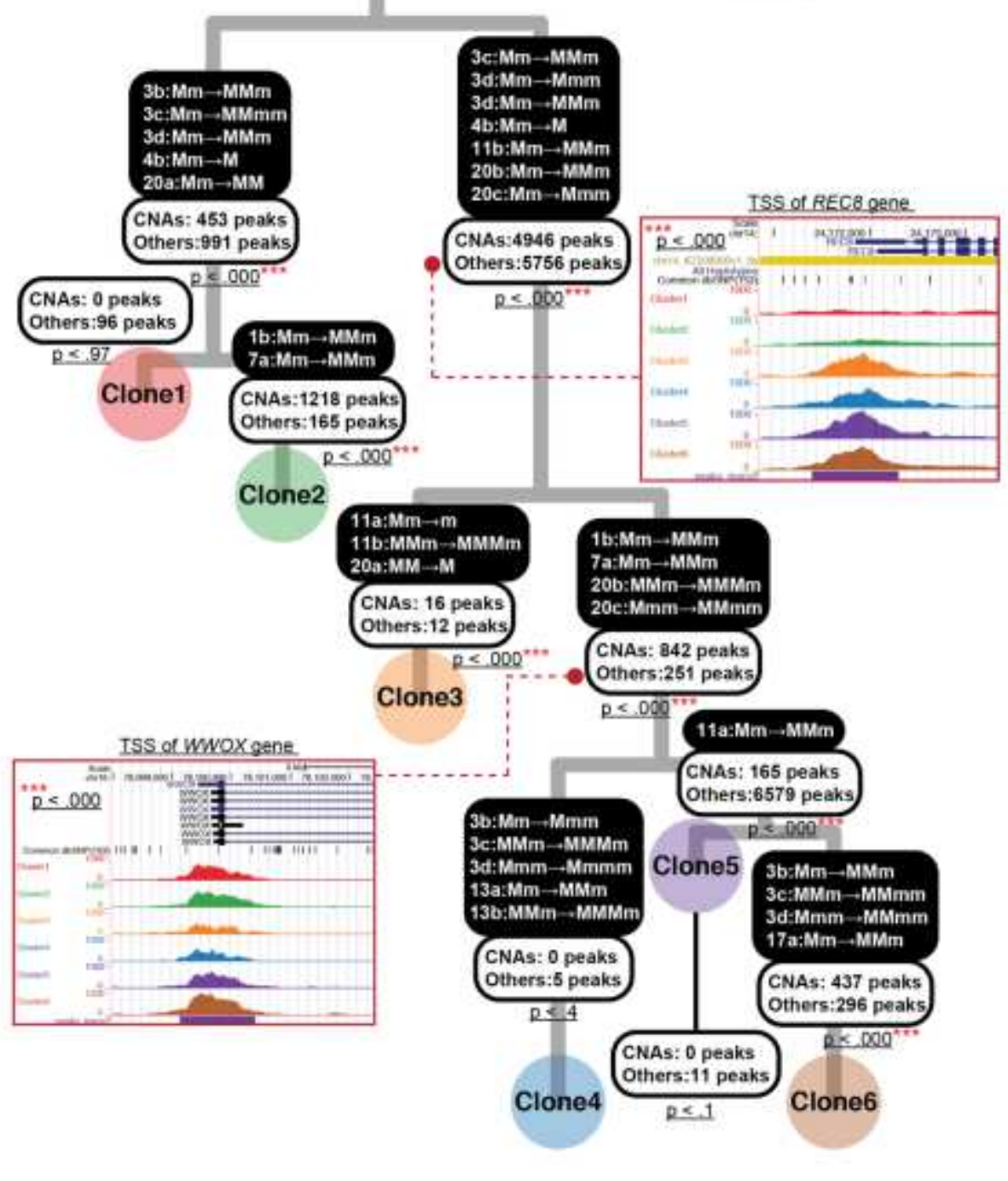

Figure 6

Integrative analysis of allele-specific copy number and chromatin accessibility for SNU601 ATAC sequencing data. (a) UMAP projection of genome-wide scATAC-seq peak profile on 2,753 cells. The same group of cells were clustered into one of the six subclones based on their allele-specific copy number profiles across the 10 selected regions. Cells in different subclones are labeled with different colors, using the same color scheme as that for the subclone labels in Fig. 4c. The number of cells colored in each UMAP is shown at the bottom-right corners. (b) A highly probable lineage history of SNU601, with copy number alterations (CNAs) and differential accessibility peaks (DAPs) marked along each branch. Pvalues of the tests for association between DAPs and CNAs are shown along each branch. For two example DAP genes, pooled peak signals for each subclone are shown as inset plots.

\section{Supplementary Files}


This is a list of supplementary files associated with this preprint. Click to download.

- SupplementaryAlleloscope.pdf

- SNU601DAPs6clones.xIsx 